\title{
Nuclear Research With Heavy Ions
}

Annual Progress Report

For the Period January 1, 1991 - December 31, 1991

Morton Kaplan

Principal Investigator
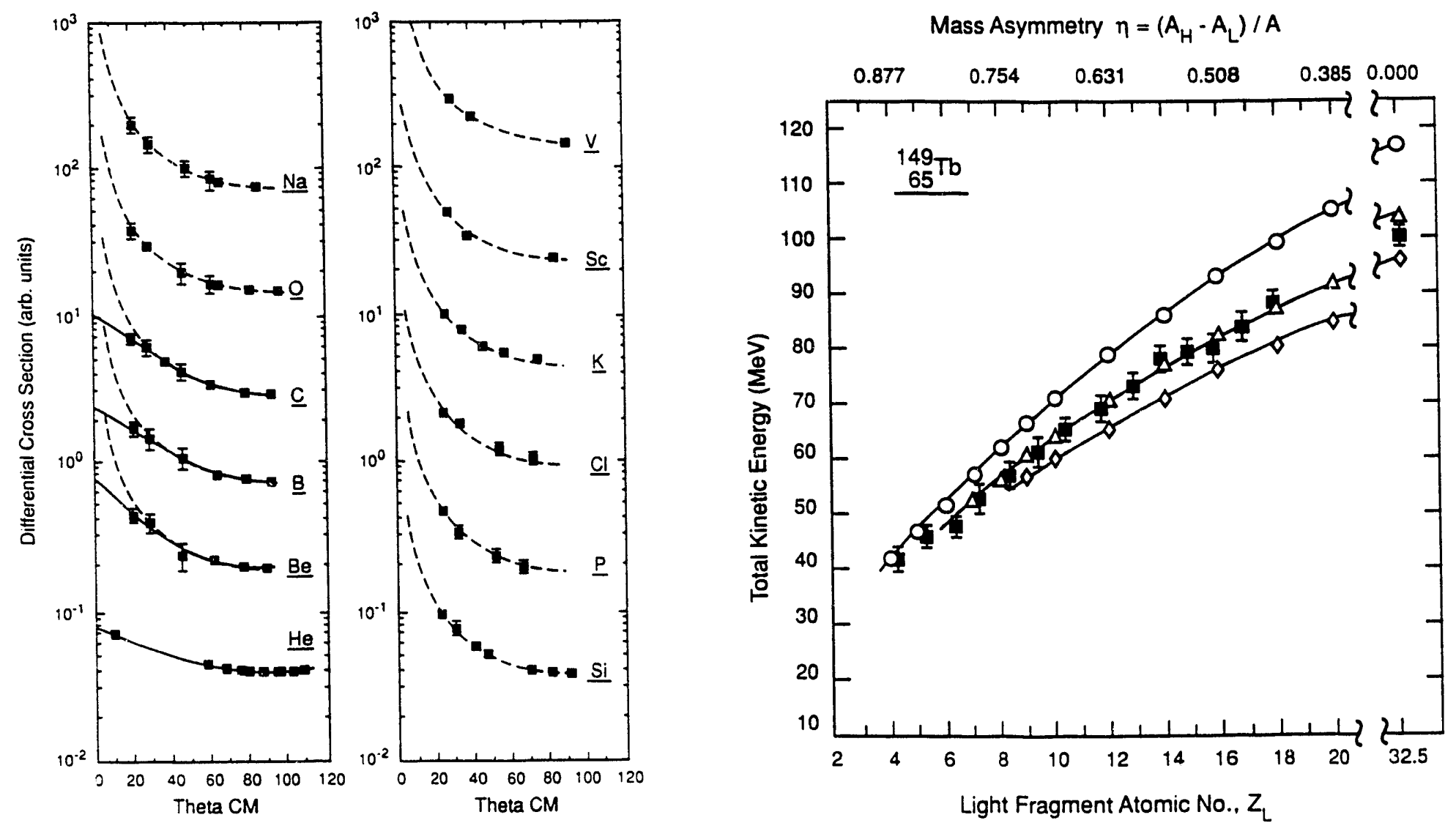

Department of Chemistry

Carnegie Mellon University

Pittsburgh, Pennsylvania 15213 


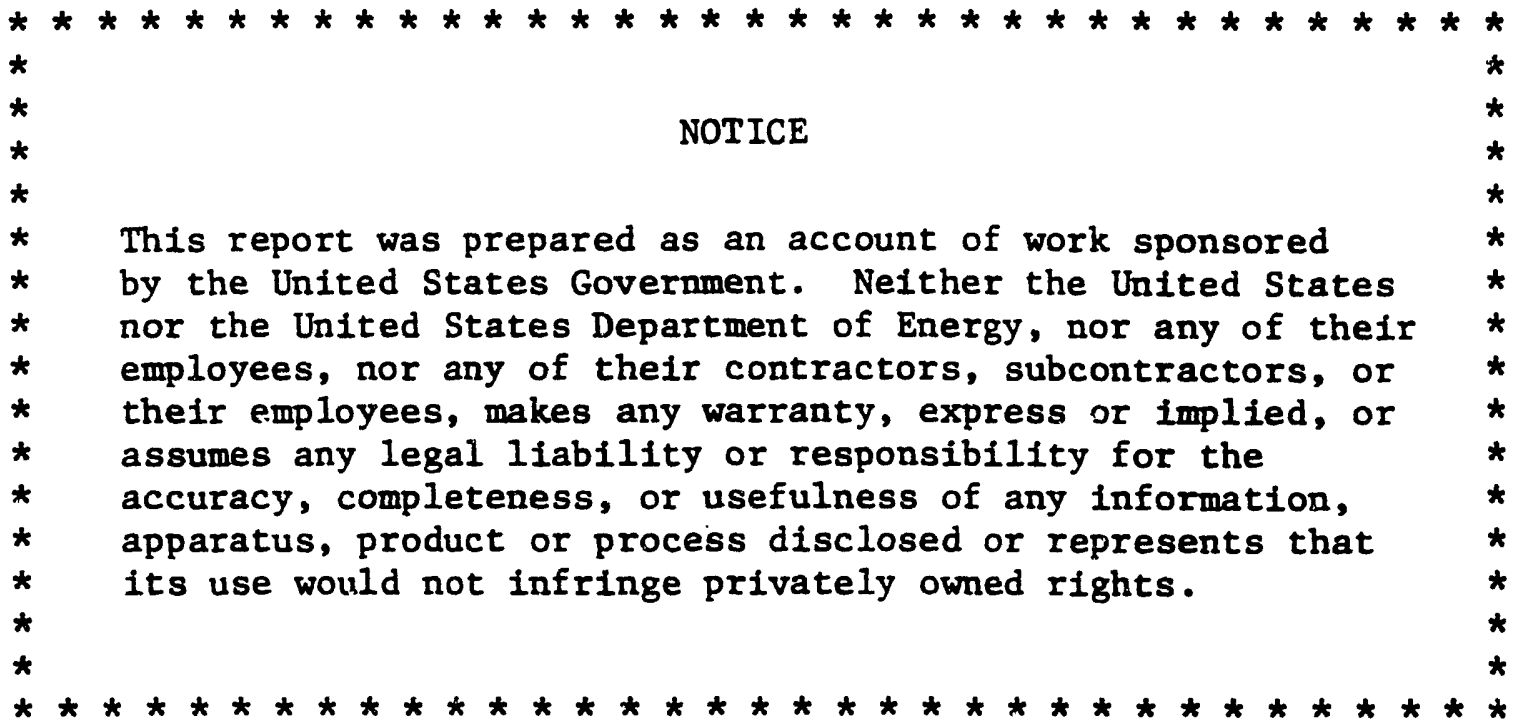




\section{TABLE OF CONTENTS}

I. Introduction $\ldots \ldots \ldots \ldots \ldots \ldots \ldots \ldots \ldots \ldots \ldots \ldots \ldots \ldots \ldots \ldots$

II. Asymmetric Fission of $149_{\mathrm{Tb}}$ * From the Finite-Range, Rotating-Liquid-Drop Model: Mean Total Kinetic Energies for

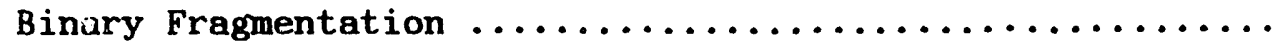

III. Charged-Particle Evaporation From Hot Composite Nuclei: Evidence Over a Broad Z Range for Distortions From Cold

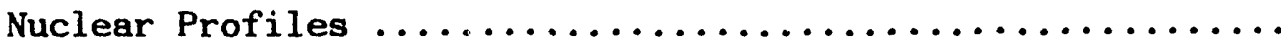

IV. The Role of Reversed Kinematics and Double Kinematic Solutions in Nuclear Reactions Studies

v. Production of Intermediate-Mass-Fragments in the Reaction $98_{\mathrm{Mo}}+5 \mathrm{I}_{\mathrm{V}}$ at an Excitation Energy $\mathrm{E}^{*}=224-\mathrm{MeV}$

VI. Emission of Light Charged Particles in the Reaction 344-MeV $28 \mathrm{Si}+12 \mathrm{l}_{\mathrm{Sb}}$

VII. Continued Developments of the Statistical Evaporation Code LILITA N90

VIII. Planning for Heavy-Ion-Collision Studies at Very High Energies: The STAR Collaboration at RHIC

IX. Scientific Personnel 95

X. Publications $\ldots \ldots \ldots \ldots \ldots \ldots \ldots \ldots \ldots \ldots \ldots \ldots \ldots \ldots$ 


\section{GRANT DE-FG0\&-87ER40324}

\section{U. S. DEPARTMENT OF ENERGY -- CARNEGIE MELLON UNIVERSITY}

ANNUAL REPORT: AUGUST 1991

\section{INTRODUCTION}

This report covers research progress and developments in the second year of the current three-year research grant supported by the Division of Nuclear Physics, U. S. Department of Energy. Our research program is experimentally based and involves studies of nuclear reactions induced by heavy-ion projectiles in complex targets over a wide range of incident beam energies. We have been concentrating our attention on measurements of light-charged particles, which we have found to be associated with almost every nuclearreaction process, and whose emission characteristics ars intimately related to reaction mechanisms and relaxation phenomena in hot nuclear matter. The overall objectives of our program are to open new avenues of approach to the understanding of nuclear behavior, especially the formation and decay of highly-excited transient states of nuclei, and to map out the evolution of nuclear reactions processes as functions of excitation energy, angular momentum, nuclear composition, and entrance channel identity. In moving towards these goals, we have over time designed, developed, and implemented a variety of experimental devices and techniques which, when used in conjunction with the predictive power of reaction-simulation computer codes, have yielded highly informative results. As much of the present efiort is part of a continuing research program, the interested reader may wish to increase his background information and/or learn of past accomplishments by referring to previous Annual Reports to the DOE in the current series. 
During the past year two major projects have been completed, namely a systematic study of charged-particle emission barriers and an extensive series of theoretical model calculations of nuclear saddle-point and scission-point shapes in highly asymmetric fission modes. The results of these investigations are presented in complete narrative form in this report. In addition, considerable progress has been made in the analyses of several recent experiments, and in our development of statistical evaporation calculations, and these are described here in more limited form as status reports. Finally, we briefly mention a sequence of actions taken this year in preparation for future research initiatives at RHIC, the Relativistic Heavy Ion Collider, now under construction at Brookhaven National Laboratory. 
IV. THE ROLE OF REVERSED KINEMATICS AND MUBLE KINEMATIC

\title{
SOLUTIONS IN NUCLEAR REACTIONS STUDIES
}

\author{
(M. Kaplan and W. E. Parker)
}

In experimental investigations of complex nuclear reactions involving heavy ions, the validity of conclusions drawn from the data frequently depends on the uniqueness with which the observables can be ascribed to specific processes. For example, measured particle spectra or angular distributions may be constituted of contributions from several emission sources or reaction mechanisms. Hence it is very important to invoke as many experimental constraints as possible, either during data collection or data analysis or both. This is especially true if comparisons are to be made with theoretical model predictions or calculations. Only by restricting the adjustability of model parameters with many interrelated or correlated measurements, can one develop definitive tests of the models and gain confidence in the physical descriptions they provide.

In studying the deexcitation of hot, high-spin nuclei, some of the more obvious experimental constraints involve coincidence measurements between heavy reaction fragments, which serve to define the reaction process, and other fragments or light emitted particles. For example, to interpret multiple sources of particle emission, it is important to measure fragment-particle correlations and make comparisons of in-plane vs. out-of-plane angular anisotropies and energy spectra. It is also very valuable to perform the same correlation studies using a reaction simulation computer code, as one does with the experimental data. Similarly, particle-particle coincidence measurements, in combination with model calculations, can be highly effective in defining the spin direction for spin-driven correlations. 
Less obvious is a special kind of constraint which arises naturally when nuclear reactions are studied using reversed kinematics. By reversed kinematics, we mean that the mass of the incident projectile is much greater than the mass of the target nucleus. To understand the origin of the process, we: must examine the differences between normal and reversed kinematics, and the effects that these differences can have on experimental measurements. In Fig. 1 we give schematic vector diagrams showing normal kinematics in (a) and reversed linematics in (b). Because of the larger center-of-mass velocity $V_{c}$ in (b), a given ejectile emission in the center-of-mass, such as $v_{1}$ or $V_{2}$, yields an increased lab velocity $V_{1 L}$ or $V_{2 L}$ in (b) compared to (a), and focusses more cross section into a forward cone. The increased lab velocity of ejectiles provides a distinct advantage of reversed kinematics, in that the effective detector thresholds are less restrictive for the measurement of ejectiles with low c.m. emission energies. Similarly, the forward focussing means that detectors can be used more efficiently in capturing a larger fraction of the emission solid angle. Not apparent solely from Fig. 1, but important in some circumstances, is the interchange of forward and backward hemispheres which occurs between (a) and (b). Finally, in (b) we see that the c.m. velocity $V_{3}$ results in ejectile detection at the same lab angle $\theta_{L}$ as $v_{1}^{\prime}$, but with much lower lab velocity $v_{3 L}$. This possibility for double solutions in the reaction kinematics does not exist in (a), and provides the basis for imposing an important constraint on experimental observations. The disadvantages of reversed kinematics are limitations in physical detactor space, more restricted angular resolution (in the c.m.), and an inherent kinematic cutoff and bias.

Figure 2 illustrates the utilization of the double solutions as a novel technique to distinguish the several sources of charged-particle emission in 
the reaction $1030-\mathrm{MeV} 121_{\mathrm{Sb}}+{ }^{27} \mathrm{Al} \longrightarrow$ fission $+{ }^{4} \mathrm{He} / 1_{\mathrm{H}}$. This reaction has very strongly reversed kinematics, and hence the two solutions for fission fragments are widely separated in energy at forward laboratory angles. The detector configuration is shown in Fig. 2(a). Two gas ionization telescopes (GT) were located each at $10 \mathrm{deg}$. with respect to the $121 \mathrm{Sb}$ beam, one in the horizontal plane (IP-GT) and the second directly above the beam (OOP-GT). These GTs were used as independent heavy fragment detectors, and each was operated in coincidence with six light particle Si telescopes (SST) placed as indicated in Fig. 2(a). The measured heavy fragment distribution for this reaction, gated by a ${ }^{4} \mathrm{He} /{ }^{1} \mathrm{H}$ coincidence requirement, is displayed in Fig. 2(b) as a contour map of $Z$ vs. Elab. The peak labeled F1 corresponds to fission fragments whose c.m. emission angles are in the forward hemisphere, and are detected at the GT laboratory angle of $10 \mathrm{deg}$. At higher $\mathrm{Z}$ and somewhat lower energies, we observe the deeply-inelastic projectile-like fragments, labeled DIR in Fig. 2(b). At much lower energies we find a second fission group, labeled F2. These latter fragments originate from c.m. emission angles in the backward hemisphere, which when combined with the large center-ofmass motion, are projected into the same $10 \mathrm{deg}$. laboratory detection angle (but with low lab energies). Fig. 2(c) gives a particularly clear view of the high resolution separation of these two equivalent, but uncorrelated, fission fragment groups, which arise from the existence of the two kinematic solutions in reactions with strongly reversed kinematics. We have tested the measured differential cross sections for the two fission-fragment groups F1 and F2, and found their ratio to be in good agreement with the ratio of their respective Jacobians, as expected.

By software gating the measured coincidence ${ }^{4} \mathrm{He} /{ }^{1} \mathrm{H}$ spectra with each of the F1 and F2 groups, which impart very different reaction kinematics to the 
light particles, it becomes feasible to uniquely distinguish and identify the emission sources. This technique, in combination with a reaction simulation code, yields spectra and multiplicities for ${ }^{4} \mathrm{He} /{ }^{i} \mathrm{H}$ evaporation from three sources: the detected fission fragment, the undetected (partner) fission fragment, and from the composite reaction system prior to fragment separation. This analysis is illustrated in Figs. 3 and 4. In Fig. 3 we show representative particle spectra in coincidence with fission fragments detected in the GT at 10 deg. in-plane (IP). In (a) the spectra are gated by the F1 group of fission fragments, while in (b) the F2 group provides the trigger gate. In both 3(a) and $3(\mathrm{~b})$, we have plotted ${ }^{4} \mathrm{He}$ spectra on the left and $1_{\mathrm{H}}$ spectra on the right, with each spectrum labeled by the lab detection (SST) angle. Above each set of spectra, we give a simplified vector diagram showing the fragment and particle detection angles and three circles corresponding to the average particle emission velocities from the three emission sources. These diagrams serve to develop a feeling for the overall emission pattern to be expected. At each SST angle, the coincident light particle spectra are shown as points and the curves are statistical model calculations (using the computer code GANES) for evaporation from the trigger fragment (-), the undetected partner fragment $(---)$, the composite system prior to fission (-.-.) and the sum (-). It is readily apparent that the ${ }^{4} \mathrm{He} /{ }^{1} \mathrm{H}$ energy spectra at corresponding angles in $3(a)$ and $3(b)$ sire distinctly different, due to the kinematic effects associated with gating on the F1 or F2 fragment solution. We have been able to fit both sets of data consistently with a unique choice of component contributions and parameters via the reaction simulation code, and this consistency within the highly constrained data sets gives a huge boost to our confidence in the selection of variables. 
In Figs. $4(a)$ and $4(b)$ we show sets of data and calculations which correspond to those in Figs. $3(a)$ and $3(b)$, except here the trigger fission fragments were recorded in the out-of-plane (OOP) GT detector. This changes the light particle kinematics yet again for both the F1 and F2 fragment groups, and still we get consigtent agreement with the model calculations without any variation in parameters or contributions from Fig. 3. In each case, once the calculational model parameters are chosen, the simulations reproduce the data sets for the F1 and F2 gates on both the IP-GT and OOP-GT triggers. As a further adaptation of the kinematics of double solutions, it should be possible to exercise a measure of control over the c.m. angle between F1 and F2 emissions. This can be done by changing the projectile-target combination or by adjusting the incident beam energy. In some special situations, this feature can be applied to the study of particle emissions both along and normal to a given axis, e.g., fission, without requiring the use of multiple detectors. Such an experiment will be discussed in the next section. 


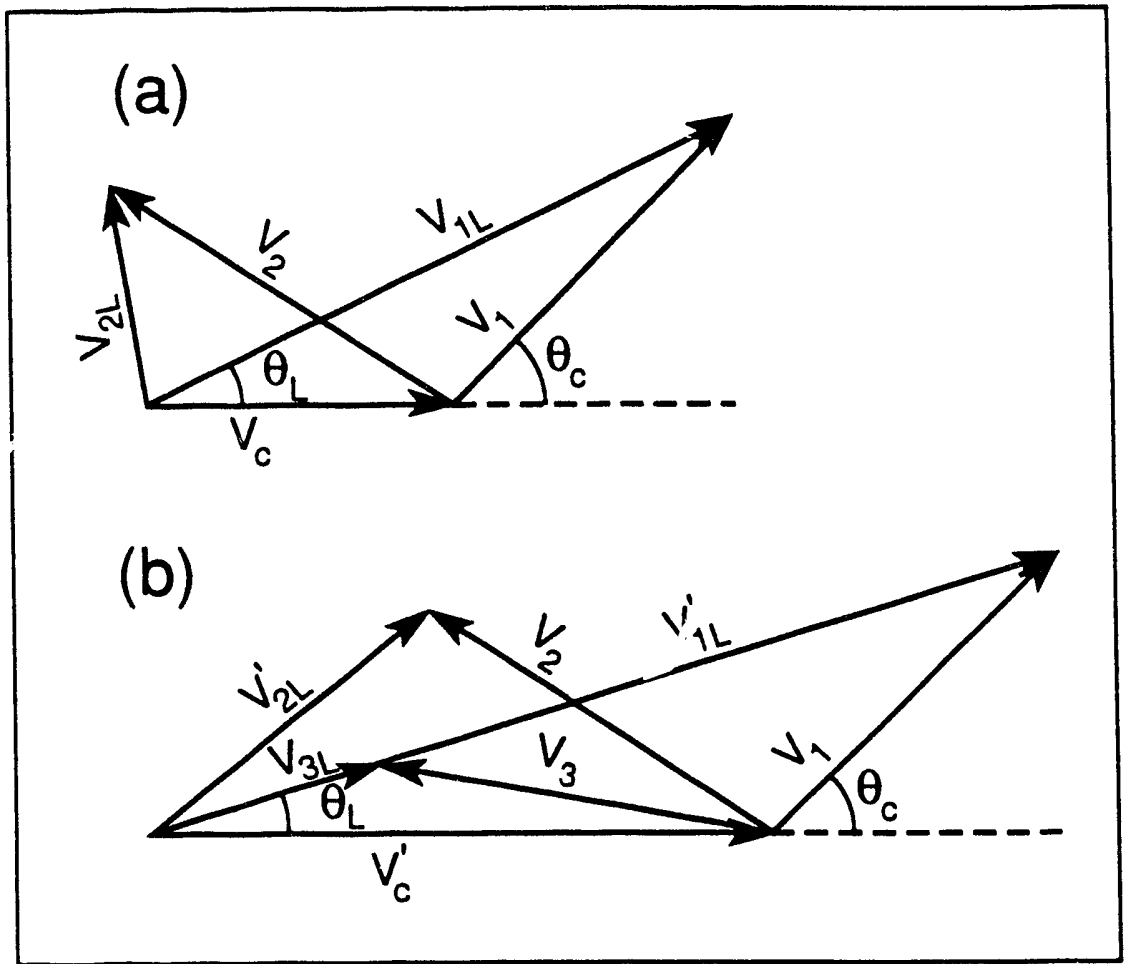

Fig. 1. (a) Normal kinematics, $M_{\text {proj }} \ll M_{\text {tgt }}$;

(b) Reversed kinematics, $M_{\text {proj }} \gg M_{\text {tgt }}$. 


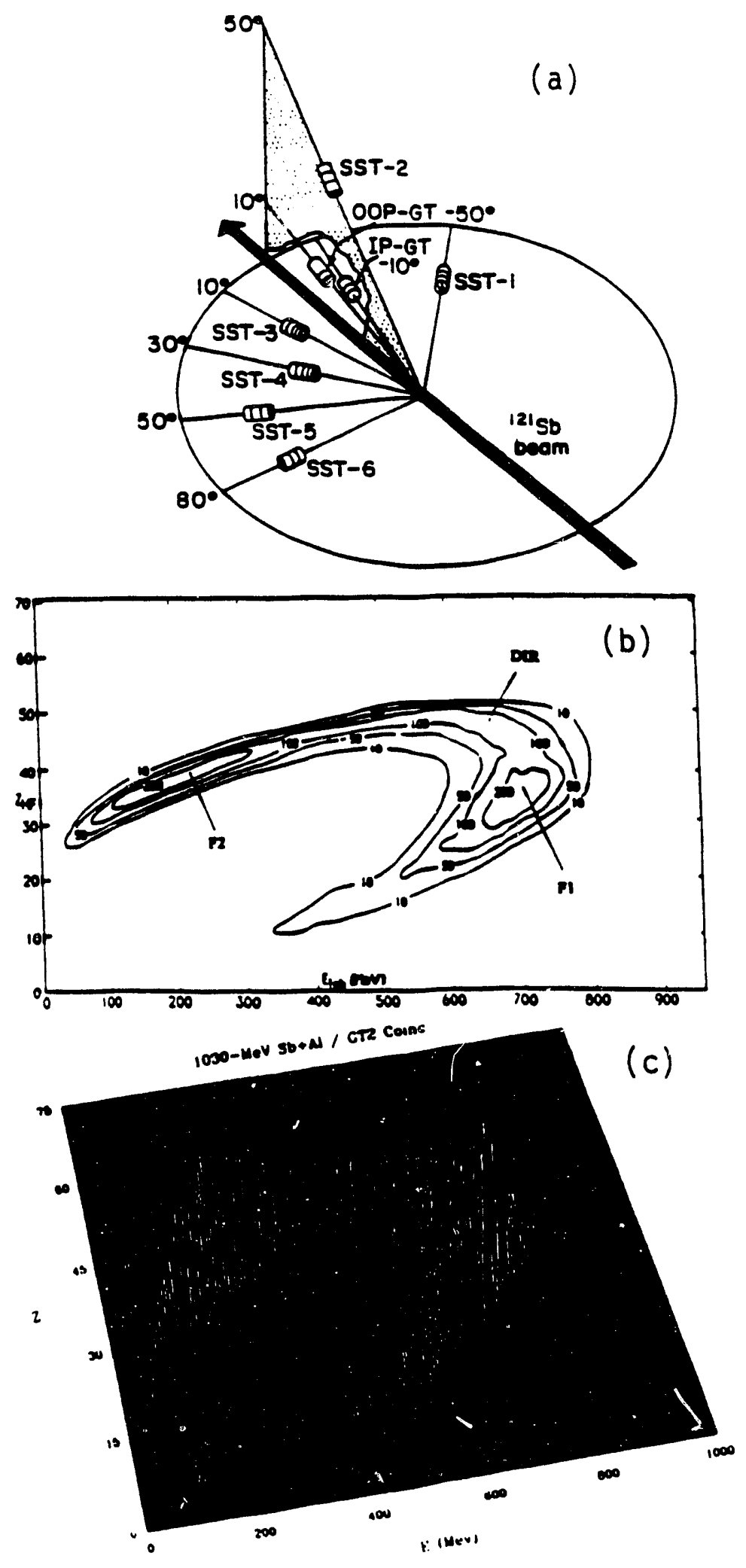

Fig. 2. (a) Detector configuration for the experiment $1030 \mathrm{MeV}{ }^{121} \mathrm{Sb}+{ }^{27} \mathrm{Al} \rightarrow$ fission $+{ }^{4} \mathrm{He} /{ }^{1} \mathrm{H}$;

(b) $Z$ vs. E contour map for heavy fragments in coincidence with a light charged particle;

(c) 3-d view of the same data as in (b). 


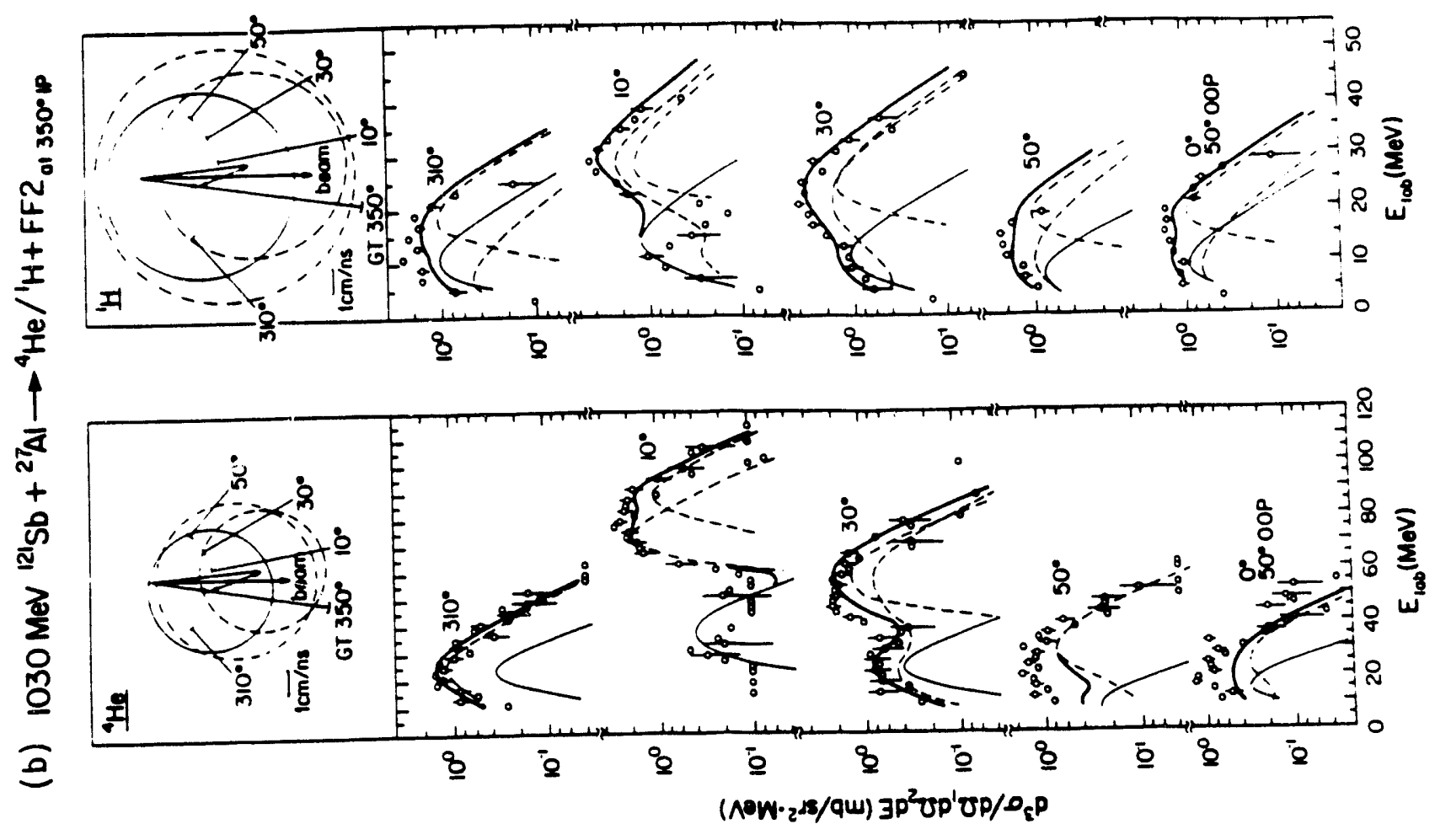

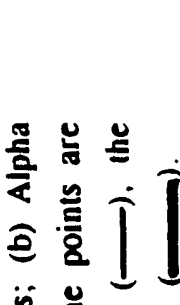
芒号  

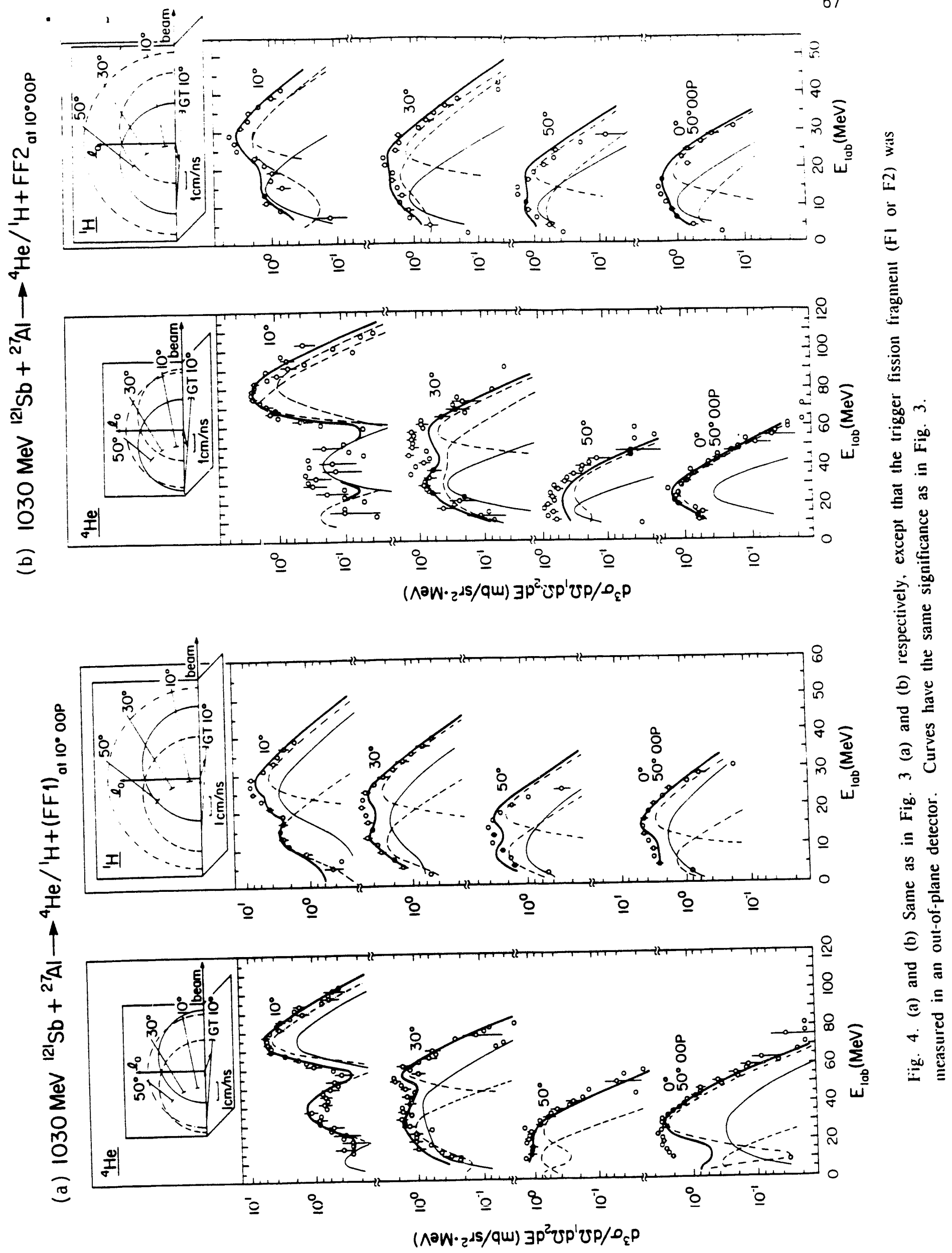


\section{PRODUCTION OF INTERMEDIATE-MASS-FRAGMENTS IN THE REACTION}

\section{Mo +51 V AT AN EXCITATION ENERGY $E^{*}=224-\mathrm{MeV}$}

(E. Vardaci and M. Kaplan)

For some time our group has studied various aspects of the formation and decay of the composite nuclear system $149 \mathrm{~Tb}^{*}$. We have now performed an experiment to measure the emission of intermediate-mass-fragments (IMF) from this highly excited system. In this work we selected the new entrance channel $856-\mathrm{MeV} 98_{\mathrm{Mo}}+51 \mathrm{~V} \longrightarrow 149 \mathrm{~Tb}^{*}$, because reaction simulations have indicated particularly interesting kinematic properties for this reaction. The reaction involves strongly reversed kinematics, but not nearly as strong as the ${ }^{121} \mathrm{Sb}+{ }^{27} \mathrm{Al}$ reaction investigated earlier (see Section IV for an example). This gives rise to two sets of fission fragment (FF) energy spectra in a single detector at accessible forward angles, corresponding to the two (unrelated) c.m. emission angles which happen by kinematics to appear at the same laboratory angle but with different energies (see Section IV). Furthermore, for the $98 \mathrm{Mo}+5 \mathrm{l}_{\mathrm{V}}$ reaction at a laboratory detection angle of 18 deg., the kinematics determines that the two FF c.m. emission angles are separated by $\sim 90$ deg. Therefore, coincidence measurements between FF and IMF (or ${ }^{4} \mathrm{He}$ ) particles will provide simultaneous observations, in the same detectors, of the correlations with respect to two orthogonal fission axes. This situation is illustrated in Fig. 1(a), where we present a kinematic vector diagram for fission and IMF emission in the $98 \mathrm{Mo}+51 \mathrm{~V}$ reaction. In this figure, $\mathrm{V}_{\mathrm{C}}$ is the velocity of the center-of-mass, and $f_{1}$ and $f_{2}$ are the velocities of two fission fragments whose c.m. emission angles and energies direct them into the same detector $(G T-2)$ at $18 \mathrm{deg}$. The partner fragments of $f_{1}$ and $f_{2}$ are undetected, and are represented by the dashed arrows, respectively. Typical IMF velocity vectors are indicated as originating from the center-of-mass (e.g., as a third 
body accompanying two fission fragments). As the c.m. emission angles of $f_{1}$ and $f_{2}$ are separated by 90 deg., it should be possible, by post-experiment software gating on the two FF groups, to compare directly the IMF (and ${ }^{4} \mathrm{He}$ ) coincidence rates in appropriately placed detectors for particles emitted along and perpendicular to the scission direction. In effect, one has the ability to rotate the fission axis by 90 deg., and comparisons can be made in the same detectors, without uncertainties between different detectors.

The actual experimental configuration is shown in Fig. 1(b). To increase our coincidence efficiency, heavy trigger (fission) fragments were detected in an array of four gas-ionization telescopes (GT), each at $18 \mathrm{deg}$. to the beam in the forward hemisphere (two in the horizontal plane containing the beam and two in the vertical plane, located above and below the beam). In addition to the GTs, the experiment employed two Wedge counters, each consisting of five $\mathrm{Si}$ stopping detectors spaced at $10 \mathrm{deg}$. intervals, with a common gas-ionization chamber for $\Delta E$ measurements. These counters were symmetrically placed to subtend the angles $38-78 \mathrm{deg}$. on either side of the beam in the horizontal plane. Because of the gas $\triangle E$ section, these Wedge detectors had rather low detection thresholds, permitting energy spectral and angular distribution measurements of IMF emissions (as well as ${ }^{4} \mathrm{He}$ particles) to be made down to relatively low energies. Each of the output signals from the Wedge counters and the GTs was split and directed to two separate amplifiers operated at high and low gain, respectively. In this manner, the experiment had sufficient dynamic range to record fission fragments, IMFs, and alpha particles in each of the detectors.

Some of the results from this experiment are shown in Figs. 2-4. Figure 2 gives the measured, inclusive, center-of-mass angular distributions for selected elements (characterized by $Z$ identification using $\triangle E-E$ analysis 
techniques) in the IMF region. For comparison, we show similar data obtained for ${ }^{4} \mathrm{He}$. The dashed curves are $(\sin \theta)^{-1}$ distributions and the solid curves are polynomial fits to the data. As can be seen in Fig. 2, all of the angular distributions are highly anisotropic, with the degree of anisotropy increasing with ejectile mass for the lighter elements. For oxygen and heavier elements, the $(\sin \theta)^{-1}$ distributions represent the experimental data quite well, whereas significant deviations are observed at small angles for the lighter elements He through C. This behavior is just what would be expected for statistical ejectile emisson from a thermally equilibrated nuclear system with high spin (i.e., the angular correlations are spin-driven). We have integrated the differential cross sections using the measured angular distributions (as in Fig. 2), in order to derive the total inclusive cross sections for production of the elements He through V. (A small low-energy shoulder which appears in the energy spectra of the elements $Z=3-20$ has been excluded from the data in Figs. 2 and 3(a). Inclusion of the low-energy contribution would increase the total integrated IMF cross section by $6 \%$.) These results are displayed in Fig. 3(a). The overall trend (after $\mathrm{He}$ ) is a smooth increase in cross section with increasing $Z$, perturbed by an enhanced yield at $C(Z=6)$ and a diminished yield at $F(Z=9)$, presumably as a consequence of the respective nuclear binding energies. We have arbitrarily considered IMFs to be in the range $Z=$ 3 - 23, as we have operationally defined the fission fragment region to be $\mathrm{Z}=$ 24 - 40. It should be clear, however, that there is no discontinuity in crossing from one region into the other. We summarize in Table I the integrated cross sections for ${ }^{4} \mathrm{He}$, IMFs, and fission. 
TABLE I. Inclusive cross sections for alpha emission, IMF emission, and fission.

\begin{tabular}{|c|c|c|c|}
\hline & ${ }^{4} \mathrm{He}$ & $\begin{array}{c}\text { IMF } \\
(Z=3-23) \\
\end{array}$ & $\begin{array}{l}\text { Fission } \\
(Z=24-40) \\
\end{array}$ \\
\hline $\begin{array}{l}\text { Cross } \\
\text { Section (mb) }\end{array}$ & $1172 \pm 59$ & $274 \pm 25$ & $442 \pm 40$ \\
\hline
\end{tabular}

Like the IMF angular distributions, the IMF energy spectra are characteristic of emissions from an equilibrated source. By this we mean that the IMF spectra, transformed to the c.m. system, have most-probable energies which are consistent with estimates from barrier systematics and the high energy slopes are indicative of relatively low temperatures. For the lighter fragments, there is a small (but significant) dependence of mean energy on c.m. angle, while for the heavier IMFs the mean energies are essentially independent of angle. The mean c.m. eneriges (averaged over angle) are plotted in Fifs. 3(b) as a function of $\mathrm{Z}$. The smooth trend of increasing mean c.m. energies with increasing fragment $\mathrm{Z}$ is consistent with Coulomb dominated statistical emission. The results in Fig. 3(b) provided the basic data set from which 〈TKE〉 estimates were derived for the comparisons described in Section II of this report (see Fig. 5 in Section II).

We now turn to coincidence measurements between IMFs and a fission fragment. These data comprise a subset of reactions in which at least three significant bodies are present in the final state - an IMF, a detected fission fragment, and an undetected fission fragment. Our results are presented in Figs. $4(a)$ and $4(\mathrm{~b})$. In Fig. $4(8)$ we give the IMF multiplicity (or the IMFfission coincidence cross section divided by the fission singles cross section at the same trigger angle) as a function of IMF c.m. angle. The fission fragment trigger angle was $18 \mathrm{deg}$. to the beam, in the same plane as the IMF 
detectors. The filled and open squares are, respectively, IMF multiplicities gated by $1^{\text {st }}$ and $2^{\text {nd }}$ fission fragment solutions in the 18 deg. detector. Because the absolute differential cross sections for $1^{\text {st }}$ and $2^{\text {nd }}$ fragment solutions are very different, it was necessary to compare multiplicities to have the two data sets on an equal footing. From Fig. 4(a) we can draw two conclusions. First, the excellent agreement in IMF multiplicities between the two data sets, even though the Jacobians are very different for the two fragment triggers, suggests that our analysis and implementation of the double-solution trigger technique has been carried out correctly. Secondly, within the statistical uncertainties of the present data sets, there is no distinguishable difference between IMF multiplicities gated by $1^{\text {st }}$ and $2^{\text {nd }}$ solution fission fragment triggers. This implies the absence of a significant correlation between IMF emission direction and the fission axis, at least at the current level of statistical error. A similar comparison is now being processed for the ${ }^{4} \mathrm{He}$-fission coincidence data, where the statistical accuracy of the data is considerably greater.

Figure 4(b) shows the IMF-fission correlation for a fission-fragment trigger at 18 deg. to the beam, but in a plane perpendicular to the IMF detectors. The angle Phi is the IMF detection angle with respect to the initial spin of the system (as determined by the trigger detector). The solid curve is a theoretically predicted function (based upon the statistical model), fitted to the data. From the out-of-plarie angular distribution in Fig. 4(b), we can integrate the IMF differential cross section to obtain the total IMF/Fission coincidence yield as $44.2 \pm 9.7 \mathrm{mb}$. This means that $44.2 \pm 9.7 \mathrm{mb}$ of IMF production $(Z=3-23)$ is in association with $442 \pm 40 \mathrm{mb}$ of fission $(Z=24-40)$, or, on the average, there is $0.10 \pm 0.02$ IMF produced per fission decay. Comparing the derived IMF yield associated with fission to the inclusive IMF yield, we 
see that only about $16 \%$ of the IMF production is fission related, implying that the bulk $(\sim 84 \%)$ of the IMFs originate in reactions which do not lead to two "normal" fission fragments along with the IMF.

A corresponding analysis of the ${ }^{4} \mathrm{He}$-fission coincidence results indicates that $372 \pm 100 \mathrm{mb}$ of ${ }^{4} \mathrm{He}$ are produced in fission associated reactions. Hence, on the average, 0.84 alpha particles accompany each fission event. Relative to the inclusive ${ }^{4} \mathrm{He}$ yield, we find about $32 \%$ of the total ${ }^{4} \mathrm{He}$ emission is fission related, or about twice as much as determined for IMFs on a fractional (or percentage) basis. Work is continuing on this project, and the analyses should be completed in the near future. 
$856 \mathrm{MeV} 98 \mathrm{Mo}+51 \mathrm{~V}$

\section{$E^{*}=224-\mathrm{MeV}$}

$$
\begin{aligned}
& V_{\text {proi }}=4.10 \mathrm{~cm} / \mathrm{ns} \\
& V_{c}=2.70 \mathrm{~cm} / \mathrm{ns} \\
& \left\langle E_{11}\right\rangle_{\text {lab }}=444 \mathrm{MeV} @ 18^{\circ} \\
& V_{f f}=1.15 \mathrm{~cm} / \mathrm{ns} \\
& \left\langle E_{12}\right\rangle_{1 a b}=118 \mathrm{MeV} @ 18^{\circ} \\
& \left\langle V_{\text {IMF }}\right\rangle_{C m}=2.85 \mathrm{~cm} / \mathrm{ns}
\end{aligned}
$$

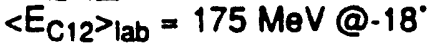

$$
\begin{aligned}
& 120 \mathrm{MeV} @+38^{\circ} \\
& 18 \mathrm{MeV} @+78
\end{aligned}
$$

(a)
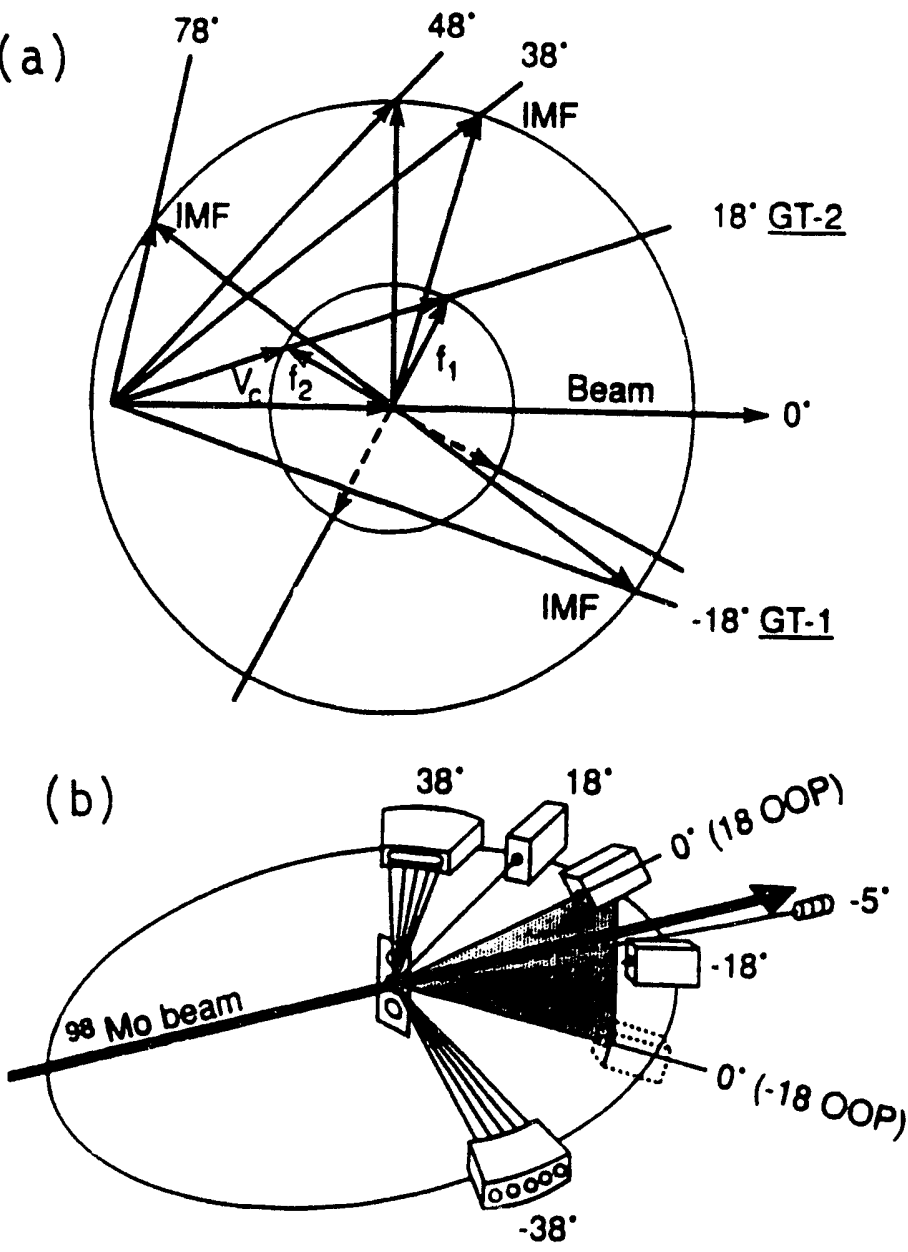

Fig. 1. Characteristics of the reaction $856 \mathrm{MeV}{ }^{98} \mathrm{Mo}+$ ${ }^{51} \mathrm{~V}$. (a) Kinematic vector diagram for fission fragments $\left(f_{1}, f_{2}\right)$ and intermediate-mass-fragments (IMF); (b) Schematic detector configuration showing four gas-ionization telescopes symmetrically located, each at $18 \mathrm{deg}$. to the beam, and two Wedge detectors covering 38-78 deg. on each side of the beam. 

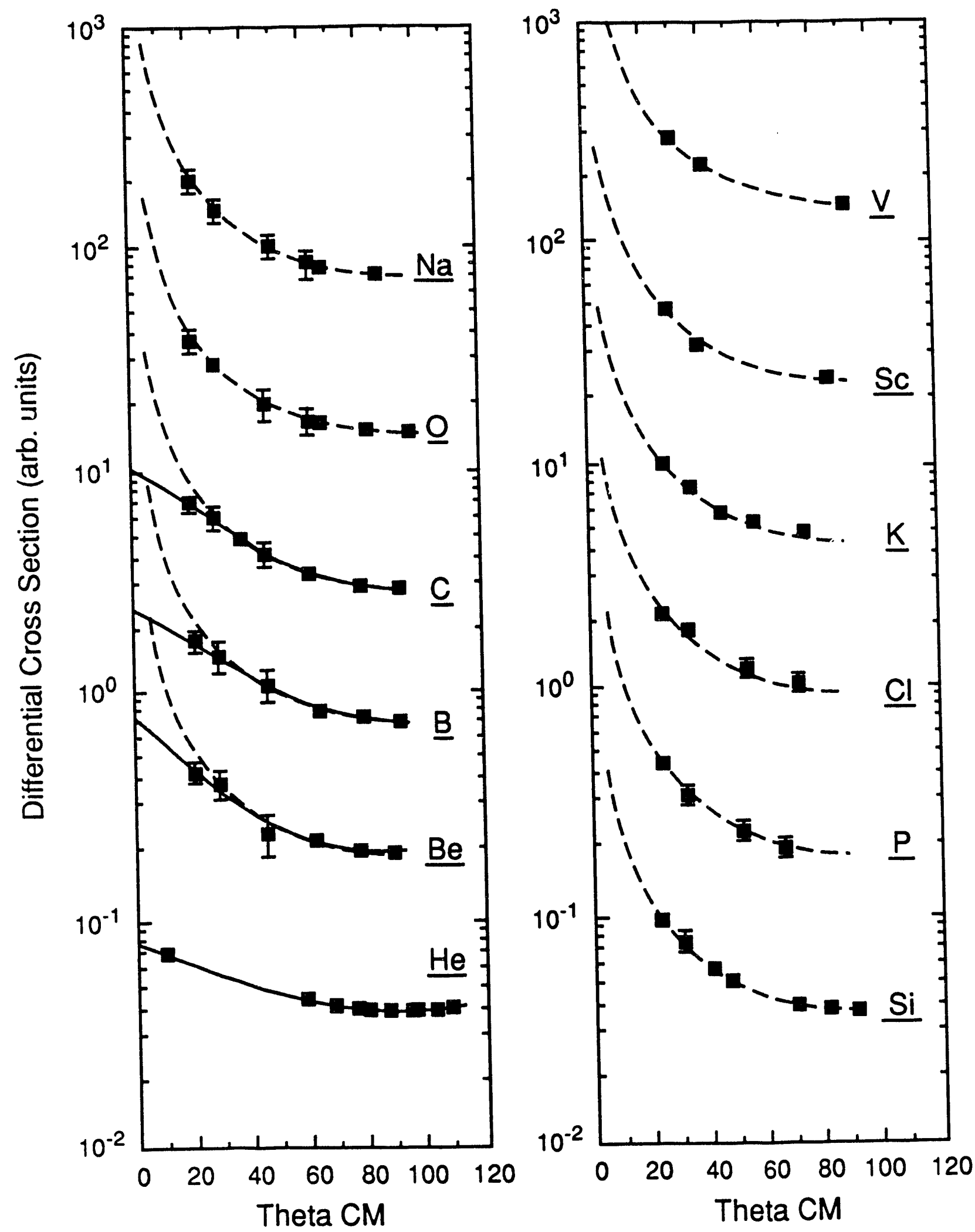

Fig. 2. Inclusive center-of-mass angular distributions for selected elements in the IMF region. The ${ }^{4} \mathrm{He}$ data is shown for comparison. The dashed curves are $(\sin \theta)^{-1}$ distributions and the solid curves are polynomial fits to the data. 
(a)

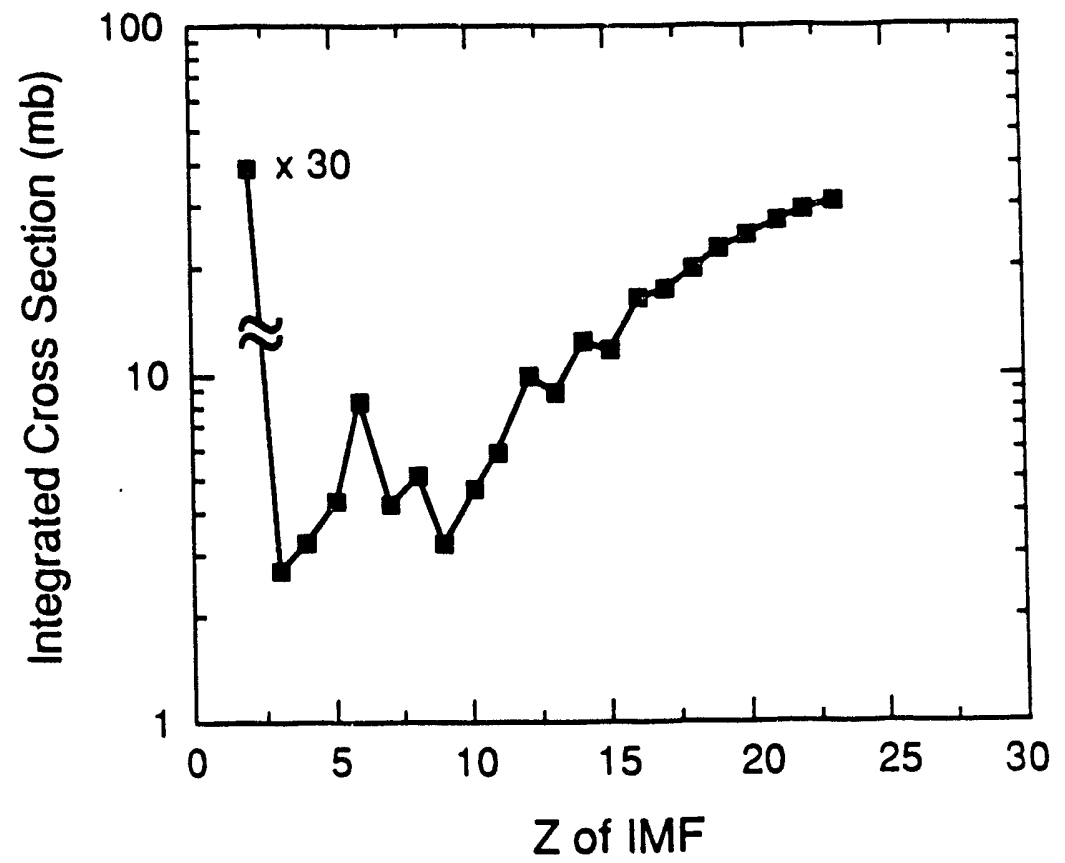

(b)

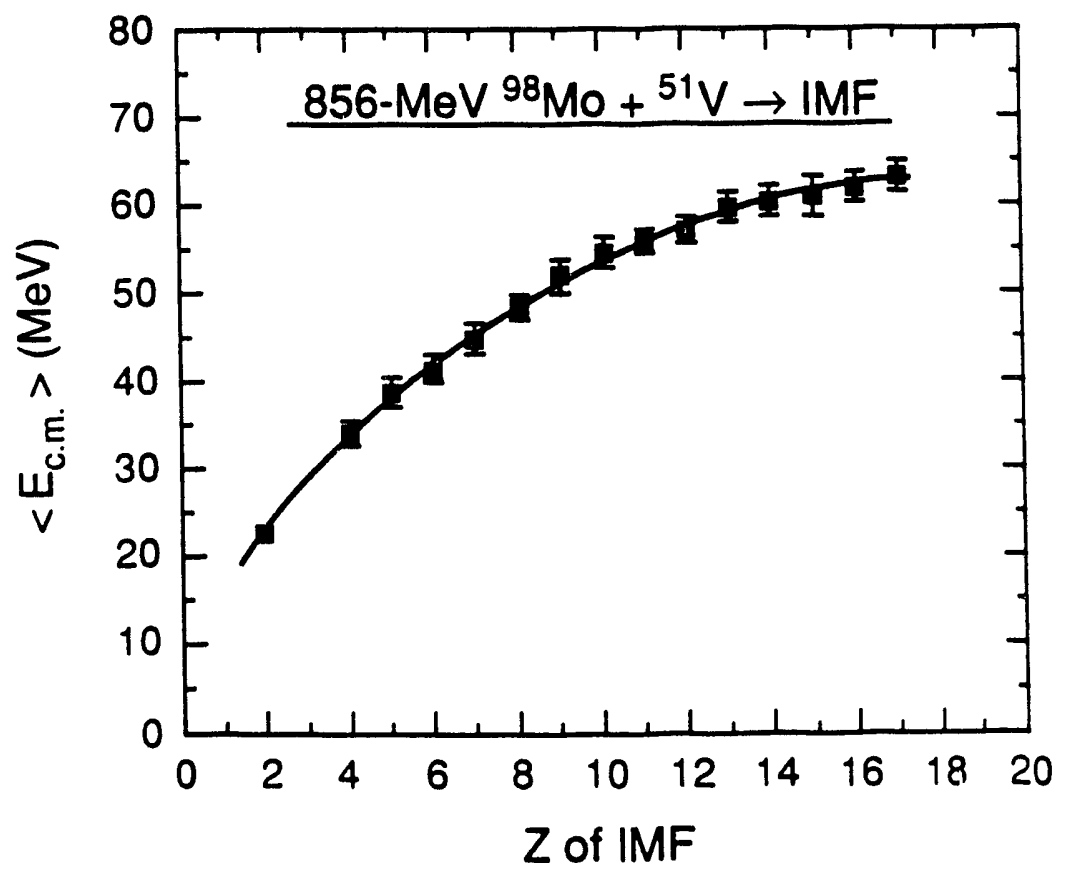

Fig. 3. (a) Integrated cross sections for elements He $(Z=2)$ through V $(Z=23)$. (b) Mean kinetic energies in the c.m. system for inclusive IMFs. 
(a)

IMF-FF Correlations

I. P. Angular Distribution $(3 \leq \mathrm{Z} \leq 23)$

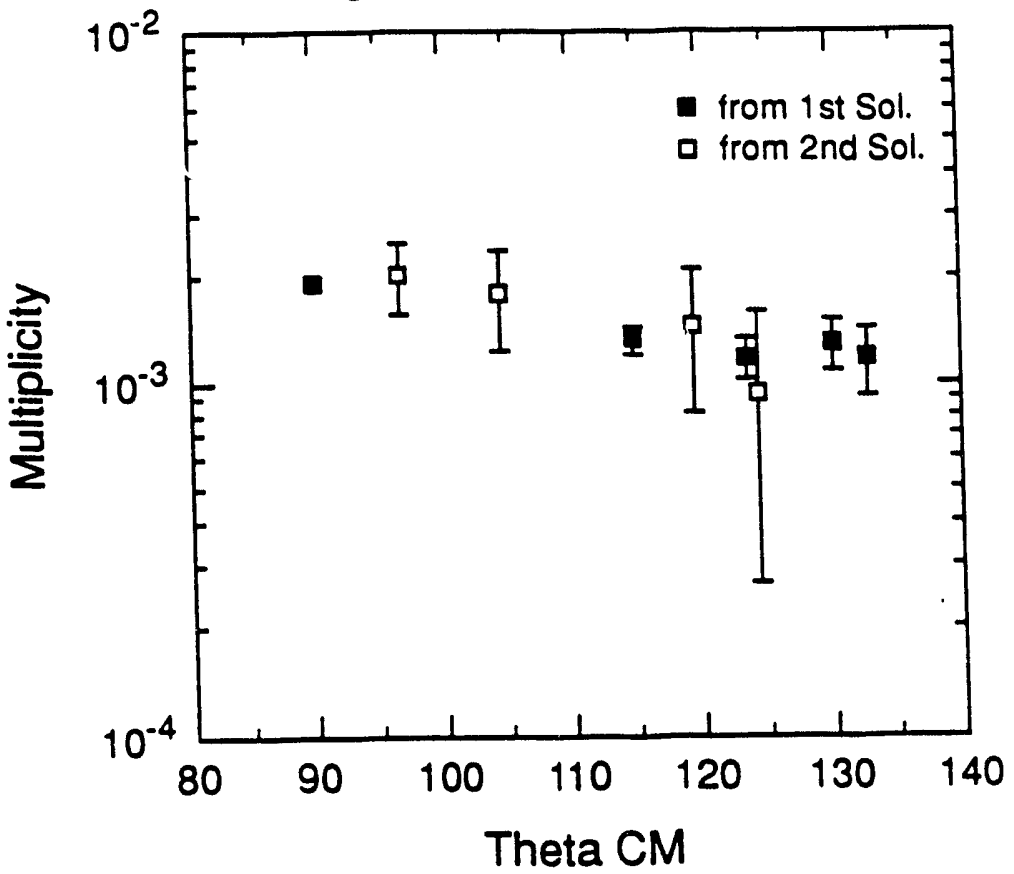

(b)

IMF-FF Correlations

O. O. P. Angular Distribution ( $3 \leq \mathrm{Z} \leq 23$ )

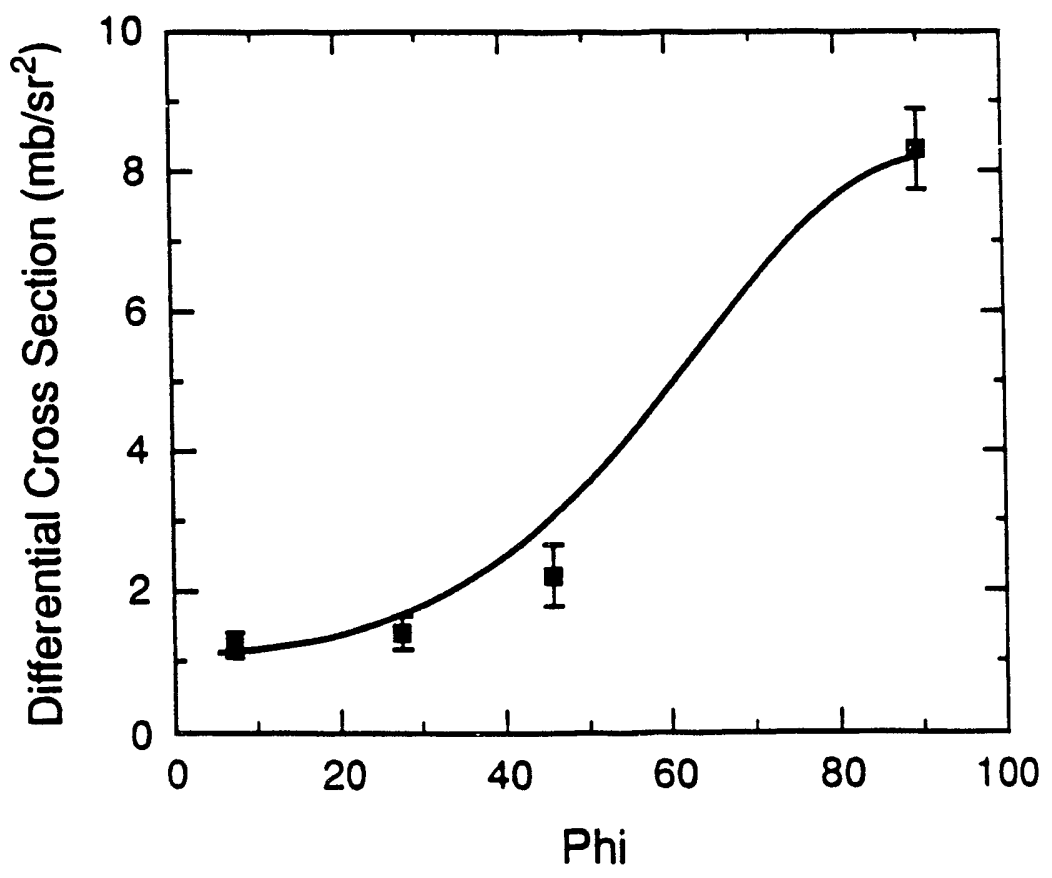

Fig. 4. (a) Differential multiplicities vs. average c.m. angle for IMFs detected in coincidence with a fission fragment at $18^{\circ}$ in the same plane. Data obtained by gating on the $1^{\text {st }}$ or $2^{\text {nd }}$ kinematic solutions for fission fragments are shown separately. as indicated. (b) Differential cross section vs, angle with respect to the spin vector for IMFs detected in coincidence with a fission fragment at $18^{\circ}$ in the plane normal to the IMF plane. The data are for $1^{\text {st }}$ solution fission fragments only. 


\title{
VI. EMISSION OF LIGHT CHARGED PARTICLES IN THE REACTION 344-MeV 28Si + ${ }^{121}$ Sb
}

(Preliminary Results)

\author{
Craig J. Copi, Paul DeYoung, and John Sarafa* \\ Department of Physics, Hope College, Holland, MI 49423
}

\author{
Morton Kaplan, Paul J. Karol, David J. Moses, Winifred E. Parker, ${ }^{\dagger}$ \\ and Emanuele Vardaci \\ Department of Chemistry, Carnegie Mellon University, Pittsburgh, PA 15213
}

Gerard J. Gilfoyle

Department of Physics, University of Richmond, Richmond, VA 23204

K. Ernat Rehm

Argonne National Laboratory, Argonne, IL 60439

\begin{abstract}
Light charged particies have been measured for the reaction $344-\mathrm{MeV} 2{ }^{\circ} \mathrm{Si}+$ $121 \mathrm{Sb}$ in singles and in coincidence with evaporation residues, fusion-fission fragments, and other light charged particles. Unique to this experiment was the use of a magnetic spectrometer in the forward direction to separate evaporation residues from the much more abundant yield of elastically scattered projectiles and projectile-like fragments. The dominant sources of evaporative 'H and "He emission are the evaporation residues (approximately 80\%), with the remainder being largely associated with fission reactions. For these latter reactions, most of the ${ }^{1} \mathrm{H}$ and " $\mathrm{He}$ can be well accounted for by evaporation from the composite system prior to fisaion and by evaporation from the postfisson fragments. Multiplicities of the light charged particles were determined for each identified emission source, and a comparison has been made to previous studies. From this comparison, indications were found for significant entrance channel effects.
\end{abstract}

\section{Introduction}

Light charged particle (LCP) emission in heavy-ion reactions has emerged as a useful tool in probing the dynamics of nuclear reactions. Ignoring forward going reactions, the three main sources of LCP are evaporation from the compound nucleus (CN), complex emission $(\mathrm{CE})$ and fragment emission (FE). The main experimental distinction of these sources is that LCP's evaporated from the $\mathrm{CN}$ are detected in coincidence with evaporation residues (ER), whereas, in the other two types of emission, the LCP's are detected in coincidence with fusion-fission (FF) fragments. Further distinction is provided by mechanistic effects. The ICP emission allows a determination of the relative strengths of these three processes. ${ }^{1-4}$ The interplay of these three competing processes provides a probe of the details of the reaction dynamics and serves as a means of determining entrance channel effects.

Since LCP evaporation provides insight into the reaction mechanism of the composite system. it is natural to use this phenomenon to search for entrance channel effects in a 
composite system. Previous work along these same lines has already been performed. The reaction $344 \mathrm{MeV}{ }^{28} \mathrm{Si}+{ }^{121} \mathrm{Sb}$ was chosen for several reasons. This reaction creates the compound nucleus ${ }^{149} \mathrm{~Tb}^{*}$ with an excitation energy of $E^{*}=240 \mathrm{MeV}$. This work is an extension of the systems previously studied by Parker, et al. ${ }^{5}$ In that study, similar compound nuclei were created with excitation energies of $E^{*}=135$ and $157 \mathrm{MeV}$ from an asymmetric entrance channel similar to the one studied here and $E^{*}=240 \mathrm{MeV}$ from a symmetric entrance channel. Hence, the reaction chosen here serves to extend the study of the systematics of LCP emission as a function of the excitation energy for the asymmetric entrance channel, as well as to allow direct comparison to a similar composite system created via a symmetric entrance channel.

\section{Experimental Setup}

The $344 \mathrm{MeV}{ }^{28} \mathrm{Si}$ beam was obtained from the ATLAS facility at Argonne National Laboratory. This beam bombarded a self-supporting ${ }^{121} \mathrm{Sb}$ target. The LCP and FF fragments were detected in solid-state telescopes (SST) $(477-527 \mu \mathrm{m}, 5000 \mu \mathrm{m})$. The ER were detected in a split-pole magnetic spectrograph. The SST's were placed at backwards angles $\left(155^{\circ}, 130^{\circ}, 105^{\circ}, \pm 75^{\circ}\right)$ to avoid complications from forward going reactions. They were all placed in the horizontal plane. The magnetic spectrograph was placed at $-6^{\circ}$ in-plane. Figure 1 provides a schematic picture of the relative detector arrangements. This produced a slight bias favoring detection of LCP in coincidence with ER in the four

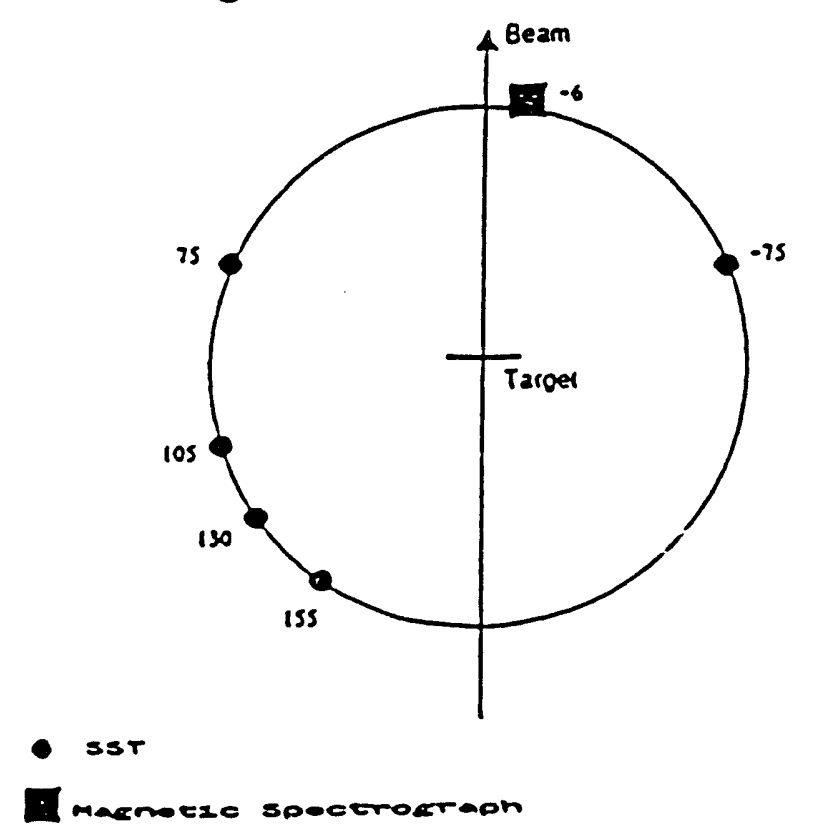

Figure 1: Relative detector arrangements showing the five SST's and the magnetic spectrograph.

SST's on the opposite side of the beam $\left(75^{\circ}, 105^{\circ}, 130^{\circ}, 155^{\circ}\right)$.

Fragment-particle and particle-particle coincidences between the SST's were recorded as well as residue-particle coincidences between an SST and the magnetic spectrograph. 
The signals from the detectors were digitized and written to magnetic tape for subsequent off-line analysis. Energy calibrations of the SST's were performed using a ${ }^{228} \mathrm{Th}$ source. Solid angles were determined by counting ${ }^{241} \mathrm{Am}$ and ${ }^{148} \mathrm{Gd}$ disintegrations from known sources placed at the target position.

\section{Inclusive Results}

Inclusive LCP and FF fragments were measured in the SST's. The resulting angular distributions for the LCP are shown in figure 2. The relatively flat distribution for $p$ and

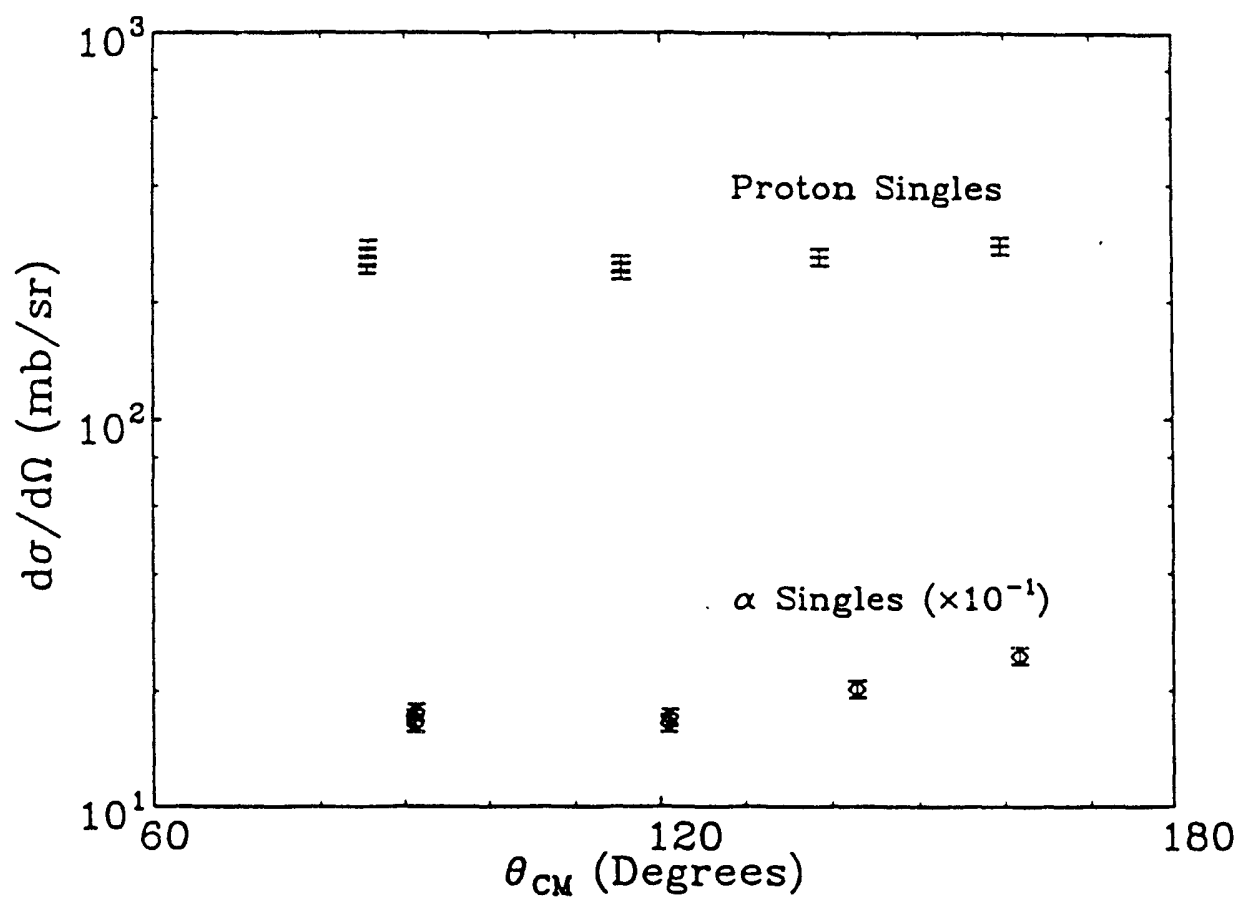

Figure 2: Inclusive $p$ and $\alpha$ angular distributions.

the anisotropic distribution for $a$ are indicative of emission governed by evaporation from the compound mucleus. The intcgrated inclusive cross sections are provided in table 1 . In

\begin{tabular}{|c|c|c|c|c|}
\hline & ${ }^{28} \mathrm{Si}+{ }^{121} \mathrm{Sb}$ & \multicolumn{2}{|c|}{${ }^{121} \mathrm{Sb}+{ }^{27} \mathrm{Al}$} & ${ }^{86} \mathrm{Kr}+{ }^{63} \mathrm{Cu}^{\mathbf{9}}$ \\
\hline$E^{*}(\mathrm{MeV})$ & 240 & 135 & 157 & 240 \\
\hline$\sigma_{p}(\mathrm{mb})$ & 3980 & 1004 & 1327 & 1666 \\
\hline$\sigma_{\alpha^{\prime}}(\mathrm{mb})$ & 2850 & 662 & 970 & 1214 \\
\hline$\sigma_{\mathrm{FF}}(\mathrm{mb})$ & 420 & 280 & 395 & 483 \\
\hline
\end{tabular}

${ }^{a}$ From reference 5 .

Table 1: Inclusive total cross sections for the LCP and FF fragments detected in the SST's.

calculating these cross scctions, it was assumed that the angular distribution for the LCP fit the form $A+B \cos ^{2} \theta_{\mathrm{CM}}$ and the angular distribution for tha $\mathrm{FF}$ fragments fit the form 
$A / \sin \theta_{\mathrm{CM}}$. Also listed in table 1 are the total inclusive cross sections for the matching symmetric and asymmetric systems. As can be seen, there is a significant difference in the LCP cross section between the system studied here and the other systems listed in the table. In all cases we have an increase in the LCP cross section. In contrast to this, the fission cross section is essentially the same for both systems with $E^{*}=240 \mathrm{MeV}$ and not very different for the lower excitation energies.

\section{Exclusive Results}

The inclusive measurements mentioned above provide some insights into various differences between the two systems under study. To gain a more thorough understanding of the processes involved in the de-excitation of the composite system, LCP-FF, ER-LCP, and LCP-LCP measurements were also made in conjunction with the inclusive measurements.

\section{A. LCP-FF coincidences}

Figure 3 shows a typical angular distribution for LCP in coincidence with a FF fragment trigger in the SST at $-75^{\circ}$. Similar angular distributions result when the FF fragment

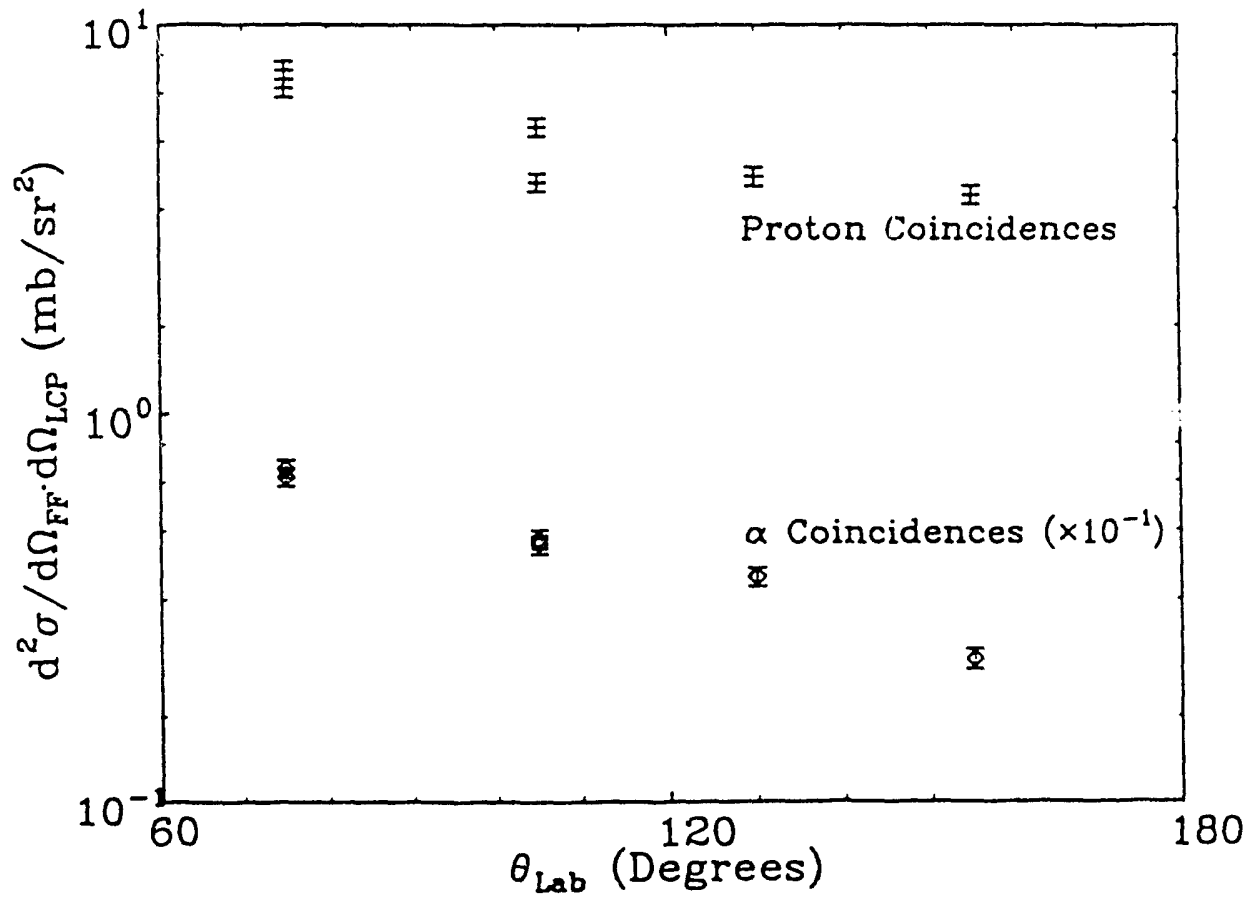

Figure 3: Angular distribution for LCP-FF coincidences in the SST at $-75^{\circ}$.

trigger is taken to be the other SST's.

To gain a more complete description of the de-excitation process, the mechanistic difference between evaporation and fission can be used to separate the sources of the light charged particles. This separation is performed via the weighted Monte Carlo reaction simulation code GANES ${ }^{6}$. The basic objective of the GANES program is the simulation of evaporative processes leading to particle emission in specified laboratory directions. By 
normalizing the simulated spectra to those experimentally observed, integrated multiplicities can be determined for each of the major sources of LCP. Typical results for $\alpha$ in coincidence with FF fragments are shown in figure 4. The figure shown here is for a FF fragment trigger in the SST at $-75^{\circ}$. The velocity vector diagram at the top of this figure shows the kinematics that lead to the separation of the LCP sources. The histograms in the spectra represent the experimental data, whereas the lines represent the separation by components as calculated by GANES using a consistent set of parameters. The thick solid line shows the overall fit to the data. In general, the calculations fit the data very well. A stringent test of the parameters was performed by doing similar fits for $\alpha$ in coincidence with FF fragments in the SST at $75^{\circ}$. The kinematics for this case are significantly different. Thus, since we attained a good fit for this case using the same set of parameters, we have confidence in the validity of the parameters chosen. The resulting calculations also fit the experimental data quite well. In a similar manner, calculations were done for $p$ in coincidence with $\mathrm{FF}$ fragments at $\pm 75^{\circ}$ using the same set of parameters. The resulting fits were of similar quality as those for the $\alpha$ coincidences and are not shown here.

With these fits it is now possible to calculated the particle multiplicities, $M$, and the LCP cross section by component. The results are shown in table 2.

\section{B. LCP-ER coincidences}

Since inclusive ER measurements were not made, the contribution to the LCP cross section from ER was calculated by subtracting the LCP contributions of CE and FE from the total inclusive cross section. These results are also listed in table 2.

Knowing the LCP-ER cross sections we can normalize the LCP-ER coincidence data. Since the LCP-ER cross sections were calculated from the singles cross section we can assume isoiropic emission of the LCP without introducing significant errors. We can then compare this result with the differential cross section from the data to find the normalization factor. For $\alpha$-ER coincidences we also correct for the fact that the $\alpha$ 's are not emitted isotropically when in coincidence with ER. The normalization factor is scaled by 1.37 as a correction. ${ }^{5}$ The $p$ 's are almost isotropically emitted when detected in coincidence with ER so we need not introduce any other correction factors in this case. With these normalizations we produce the angular distribution given in figure ??. The component cross sections now match those given in table 2 .

\section{LCP LCP coincidences}

Using the five SST's we also measured LCP-LCP coincidences. Figure 6 shows typical angular distributions for $\alpha-\alpha, \alpha-p, p-\alpha$, and $p-p$ coincidences, all triggered on the SST at $-75^{\circ}$. Assuming isotropic emission of both LCP's we can calculate the maximum LCPLCP cross sections. The results are given in table 3 along with the results from previously studied systems.

As with LCP in coincidence with FF fragments, we can also use GANES to gain more information on the processes that lead to LCP-LCP coincidences. Spectra of LCP 


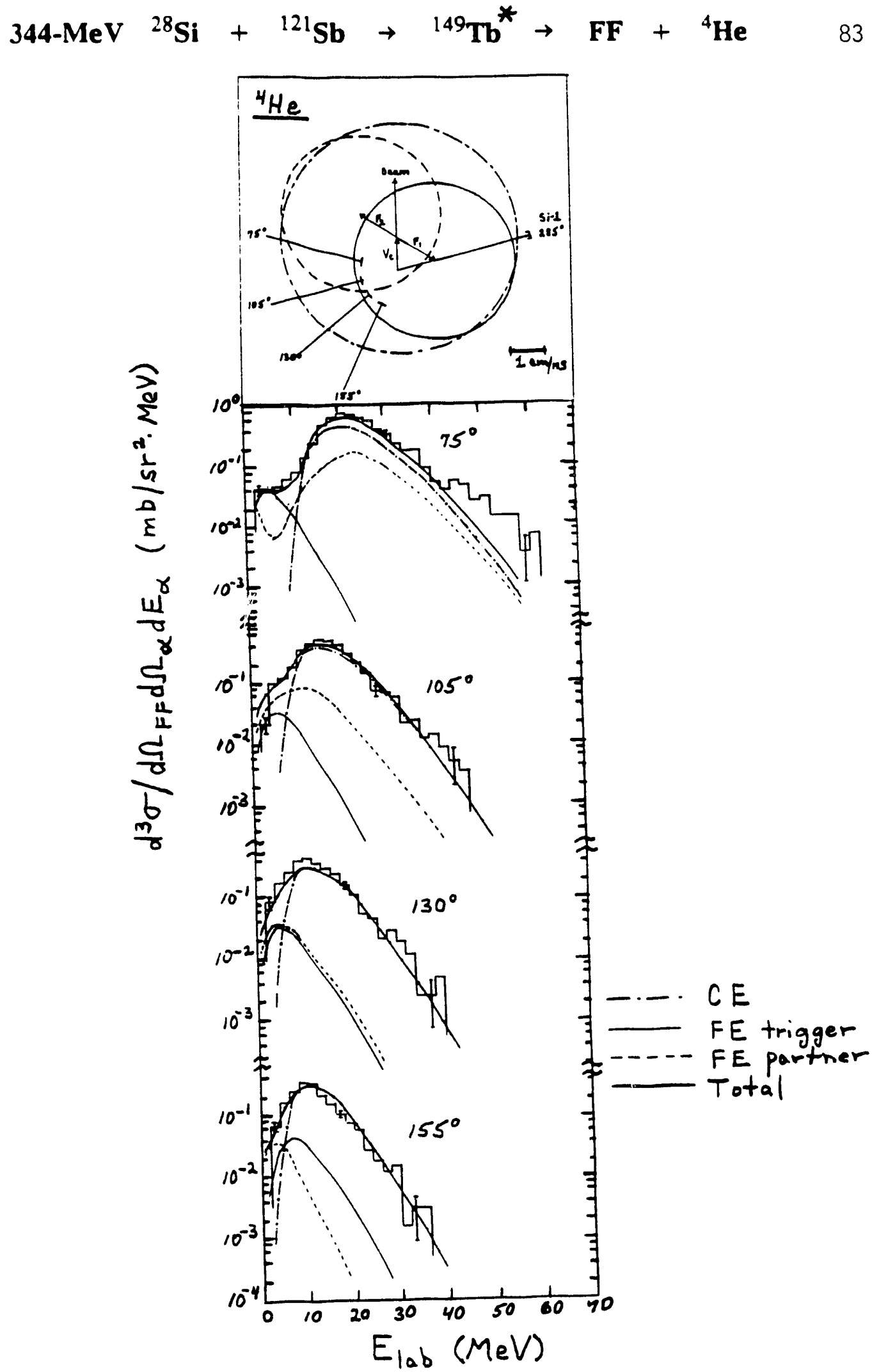

Figure 4: Spectra for $\alpha$ in coincidence with FF fragments in the SST at $-75^{\circ}$. The velocity vector diagram at top shows the kinematics involved that lead to the separation of components as seen in the spectra. The circles show the average $\alpha$ velocity from each source. 


\begin{tabular}{|c|c|c|c|c|}
\hline & $M_{\mathrm{CE}}$ & $\sigma_{\mathrm{CE}}(\mathrm{mb})$ & $M_{\mathrm{FE}}$ & $\sigma_{\mathrm{FE}}(\mathrm{mb})$ \\
\hline \multicolumn{5}{|c|}{$344 \mathrm{MeV}{ }^{28} \mathrm{Si}+{ }^{121} \mathrm{Sb}$} \\
ER-p & $-^{\mathrm{a}}$ & 3234 & 0 & 0 \\
FF-p & 1.365 & 573 & 0.42 & 172 \\
FF- $\alpha$ & $--^{a}$ & 2329 & 0 & 0 \\
\hline \multicolumn{5}{|c|}{$905 \mathrm{MeV}^{121} \mathrm{Sb}+{ }^{27} \mathrm{Al}^{\mathrm{b}}$} \\
\hline ER-p & 1.156 & 869 & 0 & 218 \\
FF-p & 0.134 & 37 & 0.231 & 65 \\
ER- $\alpha$ & 0.734 & 552 & 0 & 0 \\
FF- $\alpha$ & 0.134 & 38 & 0.130 & 36 \\
\hline \multicolumn{6}{|c|}{$1030 \mathrm{MeV}{ }^{121} \mathrm{Sb}+{ }^{27} \mathrm{Al} 1^{\mathrm{b}}$} \\
\hline ER-p & 1.409 & 972 & 0 & 0 \\
FF-p & 0.256 & 101 & 0.439 & 173 \\
ER- $\alpha$ & 1.122 & 774 & 0 & 0 \\
FF- $\alpha$ & 0.213 & 84 & 0.153 & 60 \\
\hline \multicolumn{6}{|c|}{$750 \mathrm{MeV}{ }^{86} \mathrm{Kr}+{ }^{63} \mathrm{Cu}^{\mathrm{b}}$} \\
\hline ER-p & 3.468 & 828 & 0 & 0 \\
FF/DIR-p & 0.457 & 221 & 1.276 & 617 \\
ER- $\alpha$ & 2.569 & 614 & 0 & 0 \\
FF/DIR- $\alpha$ & 0.429 & 208 & 0.812 & 392 \\
\hline
\end{tabular}

${ }^{a}$ The CE multiplicity has not been calculated.

${ }^{b}$ The results for these systems come from reference 5 .

Table 2: Comparison of the LCP multiplicities and cross sections by emitter source as determined by the fits of the GANES spectra to the data.

\begin{tabular}{|l|c|c|c|c|}
\hline & $\alpha-\alpha$ & $p-\alpha$ & $p-p$ & $\alpha-p$ \\
\hline \multicolumn{5}{|c|}{$344 \mathrm{MeV}^{28} \mathrm{Si}+{ }^{121} \mathrm{Sb}$} \\
\hline$\sigma_{\mathrm{CM}}^{\mathrm{Max}}(\mathrm{mb})^{\mathrm{a}}$ & 5347 & 6786 & 8752 & 6508 \\
\hline \multicolumn{5}{|c|}{$905 \mathrm{MeV}^{121} \mathrm{Sb}+{ }^{27} \mathrm{Al}$} \\
\hline$\sigma_{\mathrm{CM}}(\mathrm{mb})$ & 490 & 508 & 734 & 536 \\
\hline \multicolumn{5}{|c|}{$1030 \mathrm{MeV}^{121} \mathrm{Sb}+{ }^{27} \mathrm{Al}^{\mathrm{b}}$} \\
\hline$\sigma_{\mathrm{CM}}(\mathrm{mb})$ & 866 & 860 & 1268 & 948 \\
\hline \multicolumn{5}{|c|}{$750 \mathrm{MeV}^{86} \mathrm{Kr}+{ }^{63} \mathrm{Cu}^{\mathrm{b}}$} \\
\hline$\sigma_{\mathrm{CM}}(\mathrm{mb})$ & 1983 & 2094 & 3313 & 2394 \\
${ }^{a} \sigma_{\mathrm{CM}}^{\mathrm{Max}}=(4 \pi)^{2} \frac{d^{2} \sigma}{d \Omega_{\mathrm{LCP}}{ }^{2} \Omega_{\mathrm{LCP}}}$ \\
${ }^{b}$ The results for these systems come \\
from reference 5.
\end{tabular}

Table 3: Comparison of the LCP-LCP cross sections. 


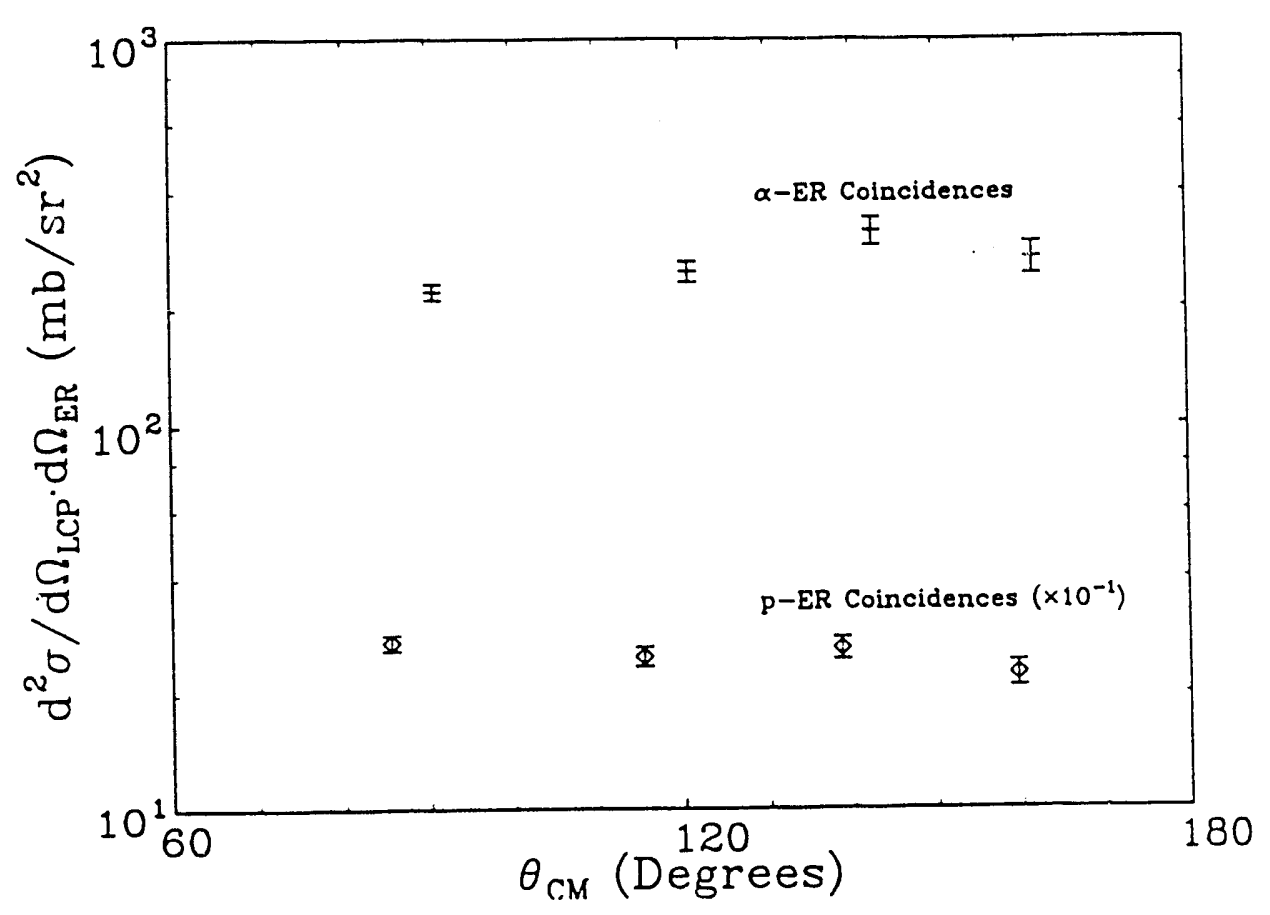

Figure 5: LCP-ER coincidence angular distributions.

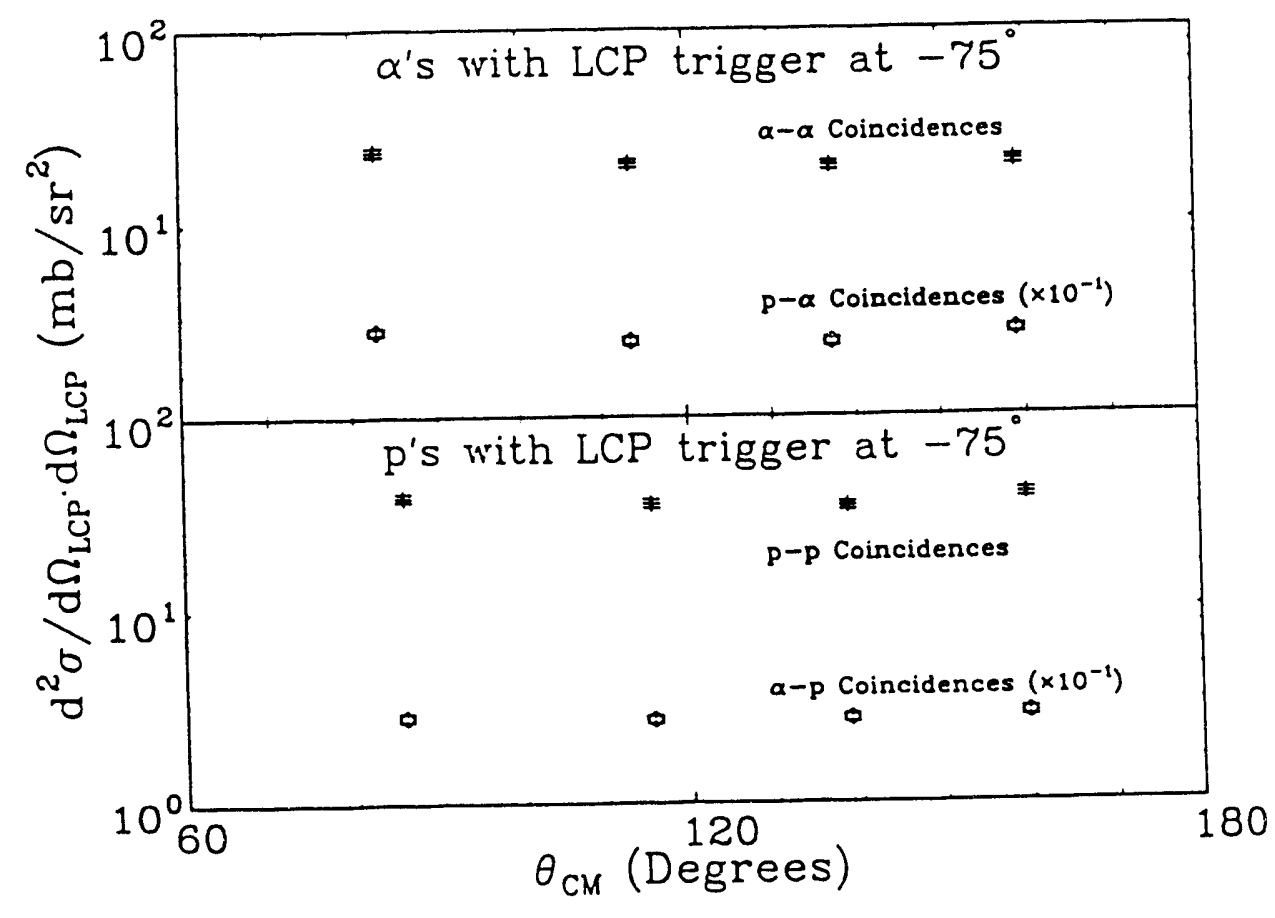

Figure 6: Angular distributions for LCP-LCP coincidences triggered on the SST at $-75^{\circ}$. 
in coincidence with LCP from CE were generated by GANES using a set of parameters similar to the set used for LCP-FF coincidences from CE. The resulting fits to the data are shown for $a^{\prime}-a^{\prime}$ coincidences in figure 7 . The solid lines in this figure are the data and
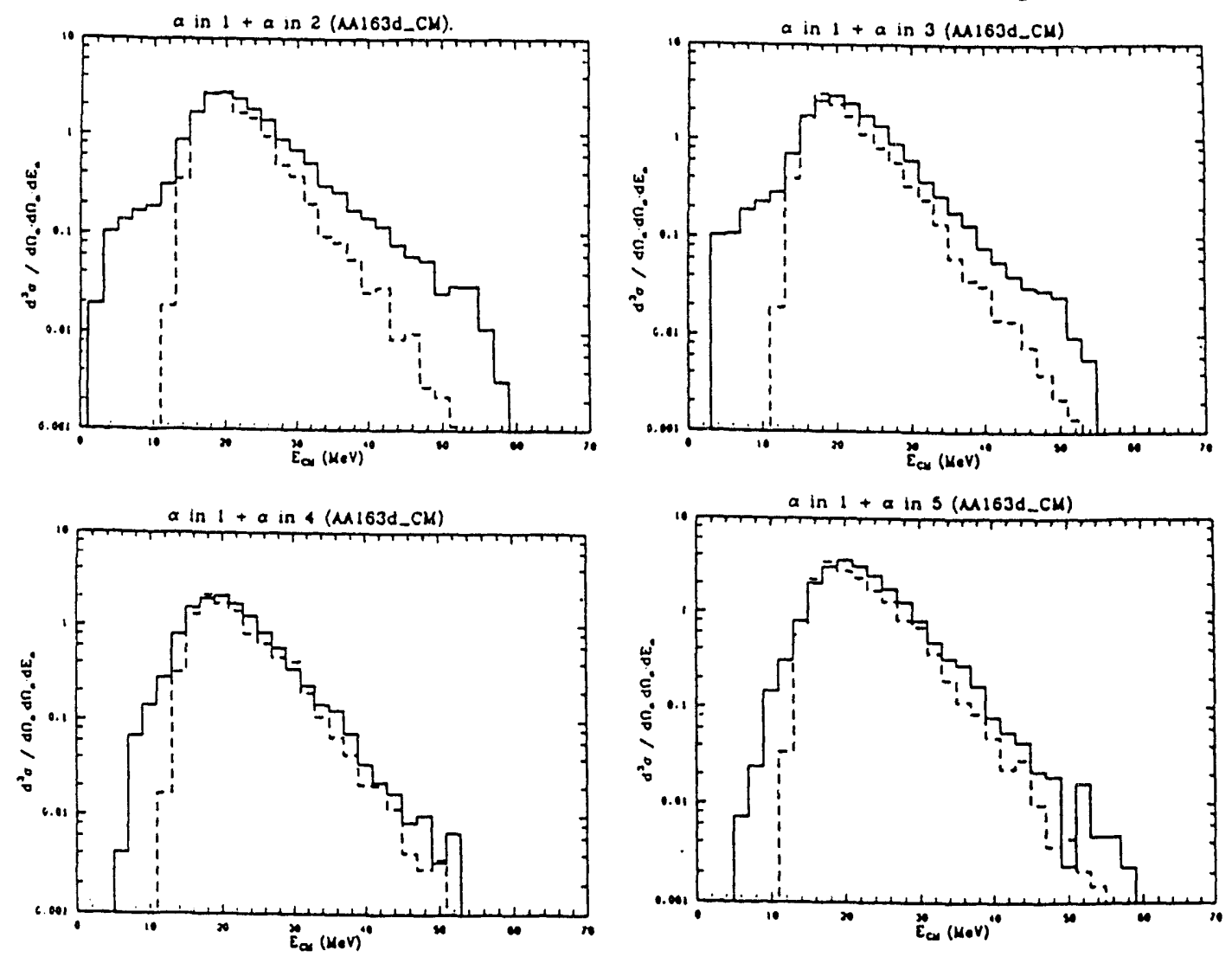

Figure 7: Spectra for $\alpha-\alpha$ coincidences showing the data (solid line) and GANES fits (dashed line).

the dashed lines are the GANES fits. From these fits we see that most of the LCP-LCP coincidence yield can be attributed to evaporation from the $\mathrm{CN}$. In the two more forward detectors $\left(75^{\circ}\right.$ and $\left.105^{\circ}\right)$ we see noticeable contributions from other sources.

\section{Conclusions}

Tables 1-3 provide concise summaries of the results to date. From these tables a number of features can be observed for the symmetric and asymmetric entrance channels. There is a substantial increase in the LCP cross sections in the asymmetric case for the system studied in this work. This is consistent with the fact that the composite system created has significantly more energy than in the two previous studies. From the coincidence data, it is observed that a large fraction of the LCP detected are evaporated from the composite system prior to fission. It is interesting to note that the $\mathrm{CE}$ cross sections from FF reactions increase with increasing energy. This result extends the trends observed in the two lower asymmetric entrance channel reactions as discussed in reference 5. 
Another interesting feature is that the CE cross sections are much smaller for both $p$ and $\alpha$ from FF reactions than for the ER production. Furthermore, the $p$ multiplicities drop more quickly than the corresponding $\alpha$ multiplicities. This observation can be explained by the fact that $\alpha$ evaporation removes more angular momentum than $p$ evaporation. Hence, $\alpha$ evaporation would provide more effective competition in high spin zone reactions such as FF reactions.

A comparison of the asymmetric entrance channel to the symmetric entrance channel shows definite indications of entrance channel effects. The significant feature to notice is that the ratio of the LCP-ER and LCP-FF cross sections for the two systems from CE shows that these types of coincidences are favored in the asymmetric case. On the other hand, the ratio of the LCP-FF cross sections from FE shows that these types of coincidences are favored in the symmetric case. This result was not observed in a comparison the similar symmetric and asymmetric entrance channels at lower energy.

The LCP-LCP coincidence work shows that most of these coincidences can be attributed to LCP's evaporated from the CN. Although there are other noticeable contributions that result from evaporation from FF fragments. The high and low energy components noticeable in the SST's at $75^{\circ}$ and $105^{\circ}$ are caused by kinematic shifts due to the motion of the FF fragments that ein t the LCP. More quantitative comparisons cannot be made until the actual LCP-LCP _ross sections are calculated by correcting the current cross sections for the fact that the LCP are not emitted isotropically. This will be done by comparison to a similar system that has information from detectors placed out of the horizontal plane.

* Present address: Department of Medical Physics, University of Wisconsin Madison, Wisconsin.

† Present address: Los Alamos National Laboratory, New Mexico.

1 L. C. Vaz, D. Logan, E. Duek, J. M. Alexander, M. F. Rivet, M. S. Zisman, M. Kaplan, and J. W. Ball, Z. Phys, A 315, 169 (1984), and references therein.

2 M.N. Namboodiri, R. K. Choudhury, L. Adler, J. D. Bronson, D. Fabris, U. Garg, P. L. Gonthier, K. Hagel, D. R. Haenni, Y. W. Li:, Z. Majka, G. Mouchaty, T. Murakami, J. B. Natowitz, G. Nebbia, R. P. Schmitt, S. Simon, J. P. Sullivan, and D. H. Youngblood, Phys. Rev. C 35, 149 (1987), and references therein.

3 R. A. Lacey, N. N. Ajitanand, J. M. Alexander, D. M. de Castro Rizzo, G. F. Peaslee, L. C. Vaz, M. Iaplan, M. Kildir, G. La Rana, D. J. Moses, W. E. Parker, D. Logan, M. S. Zisman, P. DeYoung, and L. Kowalski, Phys. Rev. C 37, 2540 (1988), and references therein, 37, 2561 (1988), and references therein.

4 D. J. Moses, M. Kaplan, M. Kildir, D. R. G. Logan, G. La Rana, W. E. Parker, R. A. Lacey, G. F. Peaslee, J. M. Alexander, N. N. Ajitanand, L. C. Vaz, and M. S. Zisman, Nucl. Phys. A465, 339 (1987), and references therein. 
5 W. E. Parker, Ph.D. dissertation, Department of Chemistry, Carnegie Mellon University, 1989 and private communication with M. Kaplan.

6 N. N. Ajitanand, R. Lacey, G. F. Peaslee, E. Duek, and J. M. Alexander, Nucl. Inst. Meth. in Phys. Res. A243, 111 (1986). N. N. Ajitanand, G. La Rana, R. Lacey, D. J. Moses, L. C. Vax, G. G. Peaslee, D, M, de Castro Rizzo, M. Kaplan, and J. M. Alexander, Phys. Rev. C 34, 877 (1986) 
VII. CONTINUED DEVELOPMENTS OF THE STATISTICAL

EVAPORATION CODE LILITA-N9O

(M. Kaplan, J. Downer, G. La Rana)

In last year's Annual Report, DOE/ER/40324-4, we described a series of major changes which we have made in the statistical-model evaporation code LILITA, originally developed at Oak Ridge National Laboratory. These important modifications dealt primarily with light particle $(n, p, \alpha)$ emission, and involved extensive revisions in the physics of the code as well as in various aspects of the computational methods employed. The revised version was named LILITA_N90 to clearly distinguish it from previous versions, and several subsidiary programs were introduced to generate master data tables and to perform various sorting functions using the expanded event-by-event file created in the running of LILITA-N90.

During the current year, the process of development and testing of LILITA-N90 has been continued. The most significant changes incorporated this year involved restructuring the manner in which numerical integrations were performed to avoid fluctuation problems in the calculated particle spectra. By replacing integrations over the variable $\left(2 \mathrm{aE}^{*}\right)^{\frac{k}{2}}$ by direct integration over the channel energy, the precision of the code was significantly improved and the resulting particle spectra were much smoother. In addition, modifications were made in the handling of the rotational energy, in order to be more consistent in the physics of the code. Because of these developments, we felt that it was necessary to repeat much of the testing which had been carried out last year, and an extensive program was initiated. The testing of LILITA-N90 is being perfomed in several stages, as outlined below. 
(1) Single step calculations were carried out for several representative nuclear systems, each involving a known initial excitation energy and a single unique spin. Furthermore, no competition was allowed between the emission of different particles, and each particle type was calculated on its own. The results of these many calculations could be compared directly with corresponding results from DOSSEM2, a much more limited, but transparent, code developed by us specifically for such testing purposes. Tests were conducted for several spin values.

(2) Having achieved a satisfactory level of agreement in (1) above, the unique spins in each of the systems studied were replaced by a spin distribution having the same r.m.s. value, and the calculations were repeated.

(3) Next, competition between particles was turned on and the single step calculations repeated once again.

(4) Finally, full multistep calculations were performed with complete spin distributions and competition between evaporated particles.

Most of the testing outlined above has now been satisfactorily completed. In the next phase, we will carry out comparison tests between LILITA-N90, which is a Monte Carlo statistical code, and a version of the code CASCADE which uses a grid structure methodology but has been modified to contain the same basic physics as LILITALN90. Eventually, we will begin comparisons with experimental data, which we have already collected for the several test systems studied here. 


\section{PlanNING fOR heAVY-ION-COLlision STUdies AT VERY HIGH ENERGIES: THE STAR COLIAABORATION AT RHIC}

In looking to the future for new research opportunities in the heavy ion field, we decided earlier this year to accept an invitation to join an existing collaboration in proposing a major experimental project for RHIC, the Relativistic Heavy Ion Collider now under construction at Brookhaven National Laboratory. This proposal, known as RHIC Letter of Intent (RLOI) *3, involves the design, fabrication, and implementation of a tracking TPC magnetic spectrometer for the purpose of exploring new phenomena and searching for a quark-gluon plasma in the high-energy-density collisions which will be produced at RHIC.

As an independent collaboration group had also proposed (RLOI 4 ) to investigate similar physics phenomena using a tracking TPC, the management at RHIC strongly suggested that the two groups (RLOI *3 and RLOI "5) conduct merger negotiations with the objective of forming a new joint collaboration to propose a single combined TPC experimental project.

Recently, the merger talks have been satisfactorily completed, and RLOI *3 and RLOI 5 have joined to become one collaboration with the name STAR (Solenoidal Tracker At RHIC). Attached to this report are three significant items which document the formation of the STAR Collaboration:

(1) The cover page of an Updated RHIC Letter of Intent *3, submitted on July $15,1991$.

(2) A copy of the RLOI 3 and RLOI 5 Merger Status, as of July 15, 1991; and

(3) A copy of an E-mail communication summarizing the conclusion of merger agreements and initiating plans for moving the project forward (July 31, 1991). 


\section{UPDATE OF RHIC LETTER OF INTENT \#3}

Search for a Quark Gluon Plasma and Other New Phenomena with a $4 \pi$ Tracking TPC Magnetic Spectrometer at RHIC *

\section{Experimental Collaboration:}

G. Danby, S.E. Eiseman, A. Etkin, K.J. Foley, R.W. Hackenburg, M. LeVine, R.S. Longacre, W.A. Love, T.W. Morris, E.D. Platner, A.C. Saulys and J.H. Van Dijk Brookhaven National Laboratory, Upton, New York 11979

\section{S.J. Lindenbaum}

Brookhaven National Laboratory and City College of New York

C.S. Chan, M.A. Kramer, K. Zhao and Y. Zhu

City College of New York, New York, New York 10091

M. Kaplan, P.J. Karol, and E. Verdaci

Carnegie-Mellon University, Pittsburgh, Pennsylvania 15219

A. Aprahamian, N. Biswas, U. Garg, V.P. Kenney and J. Piekarz

University of Notre Dame, Notre Dame, Indiana 16556

D.L. Adams, S. Ahmad, B.E. Bonner, J.A. Buchanan, C.N. Chiou, J.M. Clement, M.D. Corcoran, T. Empl, H.E. Miettinen, G.S. Mutchler, J.B. Roberts, J. Skeens and I. Stancu Rice University, Houston, Texas 77251

Spoikesman: B.E. Bonner

Management and Policy Board

B.E. Bonner, K.J. Foley, V.P. Kenney and S.J. Lindenbaum

* This research was supported by the US Department of Energy under Contract Nos. DE-AC02-76CH00016, DE-AC02-83ER40107, DE-FG05-87ER40309 and the National Science Foundation. 


\section{RLOI\#3 and RLOI\#5 Merger Status as of July 15, 1991}

The STAR (formeriy RLOI\#5) and RLOT*3 Collaborations have considerable uvcrlap of physics and detcetor interests. The two collaborations have entered into a joint RHIC R\&D project to detcrmine the performance of Time Projection Chambers (TPC) at RHIC, to optimize the configuration of a TPC-bascd cxperiment and to perform TPC tests. Results of this R\&D ffor describing the opcration and the performance of TPC's at RHIC were prescated in kic Open Mecting on RHIC Detectors in April at BNL. In addition, the two collaborations isave met in April and in June to discuss resulis and have entered into merger discussions as outlined in the memorandum below. A merger board was formed and has met on two occasions $w$ this date with plans for further mectings.

\section{Mcmorandum of Understanding - June 20, 1991}

Following discussions between the STAR and RLCI3 cullaborations, there is agreement that:

- there is considerable overlap in the physics guals of the two collaborations and unanimuus agreement that a large acceptance TPC spectumeter is an essential component of the RHIIC program;

- the two collaborations will work tugether to develop a new collahoration which will pursue the goal of building a large acceptance TPC spccummeler at RHIC;

- a board will be formed with equal representation from cach collaboration which is charged w reconmend an experimental layout to the two collaborations by August 9, 1991;

- the spokcspersons of RLOI3 and STAR will continuc to discuss he management structure inr the new collaboratiun, recisgnizing that agreement has not yet been reached on this important matter and that agreement must be reached by August 25, 1991 for the new collaboration to present its case to the PAC;

- a covering letter describing this proxess will be submitted with the upduted letters of intent from the two collaborations. 
SUMMARY Of RLOI\#3/STAR JOINT BOARD MEETING (Held at BNL on JUly 31, 1991)

The RLOI\#3 and STAR Collaborations are now one collaboration. The configuration will be sased upon the solenoidal design. The collaboration name will be STAR (Solenoidal Tracker At RHIC). The spokesman is John Harris. There are two deputy spokesmen, appointed by John, - Ed Platner and Art Poskanzer. A council consisting of institutional representatives, the spokesperson and deputy spokespersons was established from the present STAR council with the additions of Ed F? =tner (Deputy Spokesman), Ken Foley (BNL), Billy Bonner (Rice U.), Paul Kenney (U. Notre Dame), Sam Lindenbaum (CCNY) and Mort Kaplan (Carnegie Mellon U.). The new representatives will require approval from their constituents at the respective institutions. Since the council is so large, an executive committee of approximately 4 to 5 members from the council will be appointed by the spokesperson for advice on matters of running the collaboration. In addition, there will be a technical advisory committee which will meet regularly, consisting of project leaders appointed by the spokesperson for advice and consultation on day-to-day progress of the various projects of STAR. It is recognized that at some point the project management will establish residency at BNL. A complete management plan will be developed and submitted as part of the proposal and will be subject to approval by RHIC management.

A Council Meeting will be held at BNL on Tuesday, August 27, to ratify the decisions of the merger board and to establish by-laws for the collaboration. This is between the RHIC TAC Meeting on Monday and the PAC Meeting on Wednesday. The next full collaboration meeting will be held at BNL immediately after Quark Matter '91 on Saturday through Tuesday, from November $16-19$. 


\section{SCIENTIFIC PERSONNEL}

In carrying out the research program described in this report, we have had the opportunity to interact and collaborate with many colleagues. We indicate below the scientific personnel who have contributed directly or by close collaboration to the current research this year.

Carnegie Mellon University:

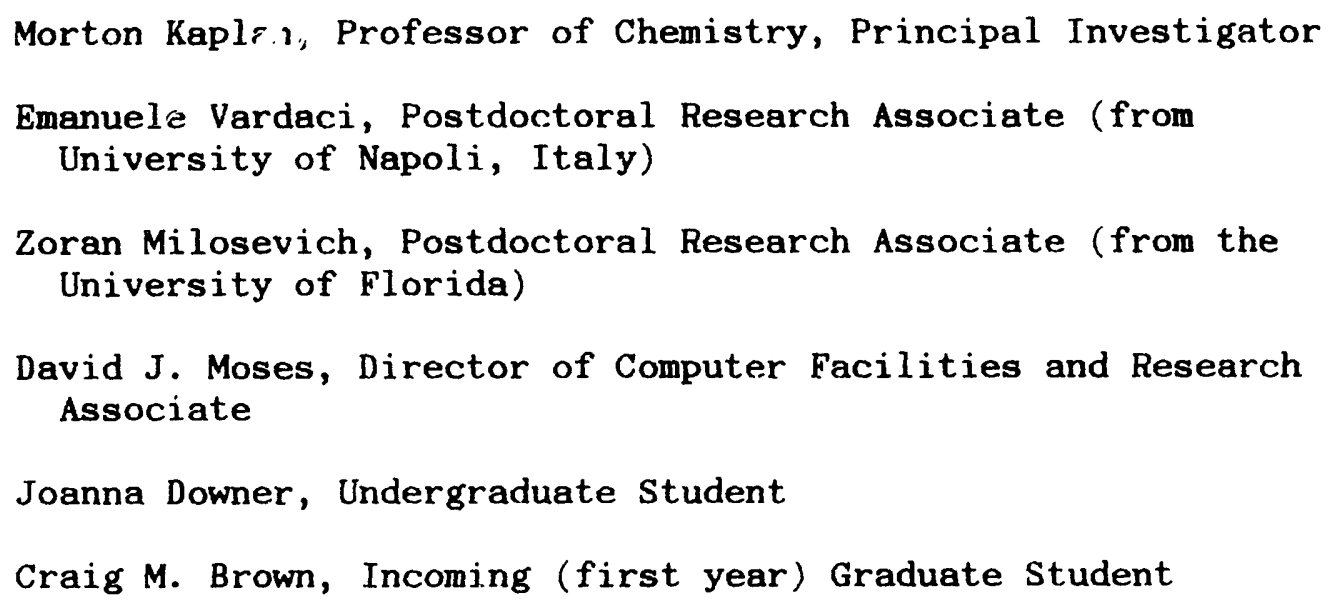

Short-Term Scientific Visitors and Collaborators:

Giovanna La Rana, University of Napoli (Italy)

Nicolae Carjan, University of Bordeaux (France)

\section{Collaborators at Hope College:}

Paul DeYoung, Associate Professor of Physics

Craig Copi, Undergraduate Research Student

\section{Collaborators at SUNY Stony Brook:}

John M. Alexander, Professor of Chemistry

Máargăet T. Magda, Research Associgte

John Boger, Graduate Student 


\section{PUBLICATIONS}

1. Charged-Particle Evaporation From Hot Composite Nuclei: Evidence Over a Broad Z Range for Distortions From Cold Nuclear Profiles. W. E. Parker, M. Kaplan, D. J. Moses, G. La Rana, D. Logan, R. Lacey, J. M. Alexander, D. M. de Castro Rizzo, P. DeYoung, R. J. Welberry, and J. T. Boger, Phys. Rev. C44, 774 (1991).

2. Asymmetric Fission of ${ }^{149} \mathrm{~Tb}^{*}$ From the Finite-Range, RotatingLiquid-Drop Model: Mean Total Kinetic Energies for Binary Fragmentation. N. Carjan and M. Kaplan, Phys. Rev. C (submitted) 1991.

3. De-excitation of Hot, High-Spin Nuclei by Particle Emission and Fission. M. Kaplan, W. E. Parker, D. J. Moses, E. Vardaci, J. M. Alexander, J. Boger, R. Lacey, and N. Carjan, Proceedings of the Seventh Winter Workshop on Nuclear Dynamics, Key West, FL 1991, J. Kapusta and W. Bauer, eds., World Scientific Publishing Co. (1991).

4. Estimation of IMF Kinetic Energies Uing the Finite-Range Rotating-Liquid-Drop Model. M. Kaplan and N. Carjan, Proceedings of the Fourth International Conference on NucleusNucleus Collisions, Kanazawa, Japan, 1991, Vol. 1, p. 127 (Report RIKEN-AF-NP-105).

5. Characterization of Charged Particle Emission in Fission By Means of High Resolution Reversed Kinematics Reactions. M. Kaplan, W. E. Parker, D. J. Moses, E. Vardaci, J. M. Alexander, and R. Lacey, Proceedings of the Fourth International Conference on Nucleus-Nucleus Collisions, Kanazawa, Japan, 1991, Vol. 1, p. 128 (Report RIKEN-AF-NP-105).

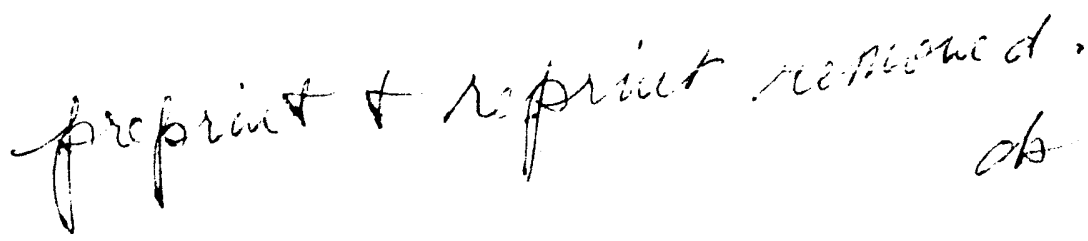



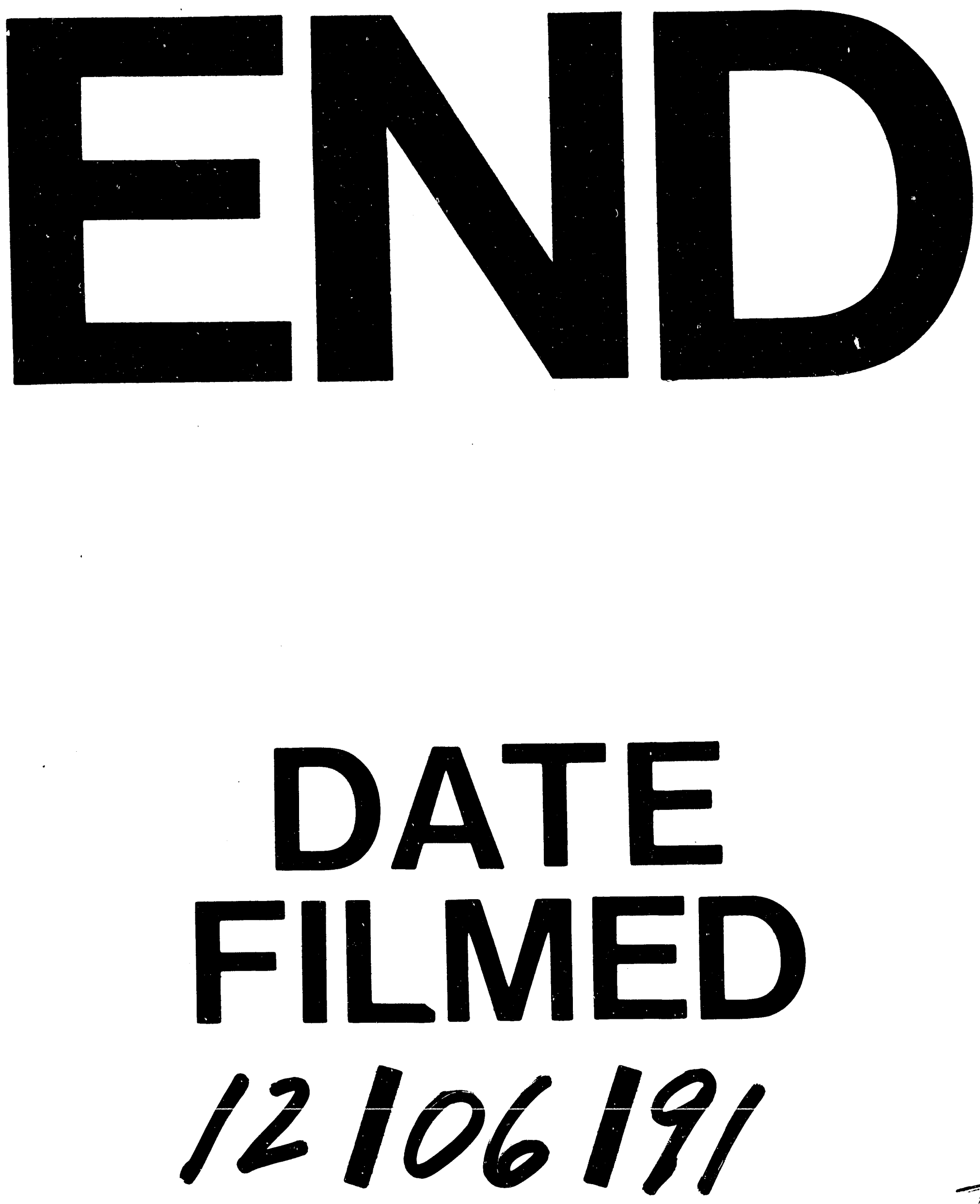

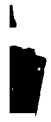


